\title{
Article \\ Dynamic Risk Assessment of Ultra-Shallow-Buried and Large-Span Double-Arch Tunnel Construction
}

\author{
Jianxiu Wang ${ }^{1,2,3, *}$, Ansheng Cao ${ }^{1}{ }^{1}$, Zhao $\mathrm{Wu}^{1}{ }^{1}$, Zhipeng Sun ${ }^{3}$, Xiao $\operatorname{Lin}^{3}$, Lei Sun ${ }^{3}$, Wuji Liu ${ }^{1}$, Xiaotian Liu ${ }^{1}{ }^{1}$, \\ Huboqiang $\mathrm{Li}^{1}{ }^{1}$, Yuanwei Sun ${ }^{1}$ and Yanxia Long ${ }^{1}$ \\ 1 College of Civil Engineering, Tongji University, Shanghai 200092, China; 2110410@tongji.edu.cn (A.C.); \\ 1830161@tongji.edu.cn (Z.W.); liuwuji@gemdalepi.com (W.L.); 2012liuxiaotian@tongji.edu.cn (X.L.); \\ 2030167@tongji.edu.cn (H.L.); 2132198@tongii.edu.cn (Y.S.); 2010328@tongii.edu.cn (Y.L.) \\ 2 Key Laboratory of Geotechnical and Underground Engineering of Ministry of Education, Tongji University, \\ Shanghai 200092, China \\ 3 Xiamen Road and Bridge Construction Group Co., Ltd., Xiamen 361026, China; \\ sunzhipeng123@hotmail.com (Z.S.); linxiaozz@hotmail.com (X.L.); sfreshair@hotmail.com (L.S.) \\ * Correspondence: wangjianxiu@tongji.edu.cn; Tel.: +86-13916185056 or +86-21-65983036; Fax: +86-21-65985210
}

check for updates

Citation: Wang, J.; Cao, A.; Wu, Z.; Sun, Z.; Lin, X.; Sun, L.; Liu, W.; Liu, X.; Li, H.; Sun, Y.; et al. Dynamic Risk Assessment of Ultra-Shallow-Buried and Large-Span Double-Arch Tunnel Construction. Appl. Sci. 2021, 11, 11721. https://doi.org/10.3390/ app112411721

Academic Editors: Dajun Yuan, Dalong Jin and Xiang Shen

Received: 12 November 2021 Accepted: 8 December 2021 Published: 10 December 2021

Publisher's Note: MDPI stays neutral with regard to jurisdictional claims in published maps and institutional affiliations.

Copyright: (c) 2021 by the authors. Licensee MDPI, Basel, Switzerland. This article is an open access article distributed under the terms and conditions of the Creative Commons Attribution (CC BY) license (https:// creativecommons.org/licenses/by/ $4.0 /)$.

\begin{abstract}
Ultra-shallow-buried and large-span double-arch tunnels face complex risks during construction. The risk sources are hidden, complicated, and diverse. The dynamic risk assessment problem cannot be solved satisfactorily by using the static method as an insufficient amount of research has been conducted. The land part of the Xiamen Haicang double-arch tunnel was selected as the background for the dynamic risk assessment of ultra-shallow-buried and large-span doublearch tunnel construction. The construction process was divided into five stages: pre-construction preparation; ground and surrounding rock reinforcement; pilot tunnel excavation; and the single-and the double-tunnel excavations of the main tunnel. Through consultation with tunnel experts, six first-level and thirty second-level risk evaluation indexes were proposed. The benchmark weight of the dynamic risk assessment index was determined by using the analytic hierarchy process. The weight of the risk evaluation index was revised according to the monitoring data and the construction stage. The fuzzy evaluation matrix of the construction risk membership degree was obtained by using the fuzzy comprehensive assessment method, and the calculation results were analyzed using the subsection assignment method. Control measures were suggested according to the risk assessment results. The risk assessment result of the double tunnel excavation stage of the main tunnel was level II, and the risk level was the highest among the five construction stages. The risk assessment result of the ground and surrounding rock reinforcement stage was level IV, and the risk level was the lowest. The dynamic construction safety risk assessment based on the fuzzy comprehensive assessment method is more timely, accurate, and reasonable than the traditional assessment method. The method can be adopted in similar engineering projects.
\end{abstract}

Keywords: ultra-shallow-buried and large-span double-arch tunnel; dynamic risk assessment; evaluation index system; analytic hierarchy process; fuzzy comprehensive assessment; risk control measure

\section{Introduction}

The construction of urban underground projects is increasing with the rapid development of urbanization. In recent years, the urban underground transportation system represented by the urban subway and highway networks has developed rapidly [1]. Double-arch tunnels have been widely used in urban underground transportation due to their small footprint, beautiful cross-section shape, and relatively small impact on the surrounding environment [2]. A double-arch tunnel uses a partition wall to connect two adjacent tunnels. This tunnel is characterized by a large excavation span, multiple construction procedures, and complex supporting structures [3]. Double-arch tunnels face various types of risks 
during the construction process, and these risks gradually develop to be hidden, complicated, and diversified $[4,5]$. In areas with complex geological conditions, tunnel excavation can easily lead to landslides, water inrush, rock burst, and other accidents [6-8], resulting in casualties and economic losses, all of which can subsequently affect the construction schedules. Risk assessment helps identify the risks related to tunnel construction, provides a reference for decision-makers to make appropriate preparations before construction, and reduces the project risks [9]. Therefore, a clear grasp of the potential risk factors is necessary at all times during the construction process, and countermeasures must be determined in advance according to the risk level.

Risk assessment calculates the degree of influence of each factor on the assessment results [10] and is a tool designed to support decision-making in all aspects, from the beginning to the end of the process [11]. Tunnel risk assessment was first proposed by Einstein $[12,13]$. Subsequently, many studies [14-16] were conducted on tunnel risk assessment, and the concept and method of tunnel risk assessment were proposed. Kuang et al. [17] analyzed the risk sources of the Xuefengshan No. 1 deep-buried tunnel and identified the main risk of tunnel construction and those in the construction process. Li et al. [18] selected twelve influencing factors from the aspects of karst hydrogeology, engineering geological conditions, and construction factors as the comprehensive risk evaluation index of karst tunnels and established a comprehensive risk evaluation index system of tunnel collapse, the large deformation of surrounding rocks, water inrush, and mud inrush. Zhang et al. [19] took the collapse risk assessment of mountain tunnels as an example and proposed a collapse risk assessment method on the basis of case-based reasoning, advanced geological prediction, and rough set theory. In recent years the soil steel structure, due to its fast assembly speed, low construction cost, continuous construction, and sustainable development, has been more extensively used in the construction of bridge, culvert and tunnel [20]. The research on the safety of the soil steel bridge, culvert and tunnel has gradually attracted the attention of scholars [21]. Maleska et al. [22,23] successively studied the durability of the soil steel bridge under rock burst and the performance of the steel soil tunnel under a seismic load. Flener's research [24] shows that the backfilling process, covering depth and sealing depth of the soil steel culvert are important factors that affect the performance of the box culvert. Common risk assessment methods include fault tree analysis, the analytic hierarchy process (AHP), fuzzy comprehensive assessment, grey clustering, and neural network. Hong et al. [25] adopted the event tree analysis method to consider the probability of accidents during construction, studied tunnel construction risks, and put forward countermeasures. Hyun et al. [26] analyzed the risk of adverse events, which may occur during the construction of a shield tunneling machine, using the AHP method and systematically assessed the overall risk level of the shield tunneling machine. Lyu et al. [27] studied the flood risk of a metro system by using the AHP and then proposed an improved trapezoidal fuzzy AHP (FAHP) to evaluate the risk related to the ground subsidence of the infrastructure in Shanghai [28]. The consistency requirements of the comparison matrix in the AHP are difficult to meet. Li et al. [29] analyzed the reasons for the inconsistency and proposed an improved AHP (IAHP), which they applied to subway construction risk identification. Chu et al. [30] selected twelve factors affecting water inrush for the evaluation index system and used the fuzzy comprehensive assessment method to evaluate the water inrush risk of a karst tunnel. Balta et al. [31] proposed a tunnel boring machine project delay risk assessment method based on Bayesian neural network. Kodu et al. [32] proposed a methodology for evaluating the important factor for the fire design of bridges. That proposed important factor was similar to those used to evaluate the wind and snow loads of buildings. Enrico et al. [33] determined important factors by comprehensively considering the concepts of hazard, exposure, and vulnerability. With the progress of society and the increasing complexity of engineering projects, the single assessment method often fails to obtain accurate assessment results. Consequently, risk assessment methods that combine two or more qualitative or quantitative analysis methods have gradually become popular. Deng et al. [34] used the historical data of fifty 
tunnels and combined the fuzzy method with a neural network to establish a tunnel risk assessment model, which they then applied to the risk assessment of the Tiefodian tunnel. Wang et al. [35] proposed a risk assessment model for coal mine flood emergency logistics by combining the AHP with a neural network. Zhang et al. [36] used the AHP method and fuzzy decision-making to evaluate the collapse risk of ridge tunnels. Lyu et al. [37] combined the interval FAHP with fuzzy cluster analysis to assess the flood risk of a subway system in a sinking environment. Lyu et al. combined the triangular fuzzy number (TFN) and the AHP into a geographic information system to assess the submergence risk of the Shenzhen subway system [38] and applied it to the evaluation of geological disasters [39].

Tunnel construction risk assessment is divided into static and dynamic risk assessments. Static risk analysis mainly focuses on the preliminary stage of the project, supporting a project feasibility study, the selection of construction methods, and the preparation of the project budget. In the construction process, the risks are evaluated and can change, and therefore the assessment results obtained by these models are often different from the actual situation. Therefore, the dynamic risk assessment of construction sites is necessary. Huseby [40] systematically introduced the principle of the dynamic risk analysis model based on the impact graph and DynRisk models of the Monte Carlo principle. At present, an increasing number of scholars are studying dynamic evaluation and have gradually studied dynamic methods after initially proposing the concept of dynamic evaluation. Currently, various methods can be used for specific and operable dynamic evaluation, and the dynamics are mainly achieved through four aspects: introduction of time and assignment of weight [41]; dynamic adjustment of the risk evaluation index system [42]; dynamic adjustment of the weights of risk evaluation indicators [43]; and division of the evaluation process into stages [44]. Wang et al. [45,46] established a dynamic collapse risk assessment method on the basis of the attributed comprehensive assessment model and evaluated the tunnel before excavation, after excavation, and before support. Wang et al. [47] proposed a dynamic risk assessment method based on a Bayesian network in view of the shortcomings of the risk assessment of deeply buried tunnels. Wu et al. [48] established a dynamic risk assessment system, including pre- and post-evaluation models, by using the attribute recognition model and performed risk analysis on sections in tunnels with a high risk of water inrush.

Few studies have been conducted on the dynamic evaluation of urban underground engineering and fewer on the dynamic evaluation of urban shallow-buried and large-span double-arch tunnels. The construction of ultra-shallow-buried and large-span double-arch tunnels is large scale and has high construction risks, and the construction process is divided into several stages. As the construction progresses, the construction environment and geological conditions of the project constantly change. Given these dynamic external factors, the risk factors vary at different construction stages, and the risk also changes and develops dynamically. To comprehensively evaluate the risks during the construction of an ultra-shallow-buried and large-span double-arch tunnel, a dynamic risk assessment of the construction of such tunnel was conducted. As such, the ultra-shallow-buried and largespan double-arch tunnel in the land part of the Xiamen Haicang undersea tunnel in China use selected. The construction process was divided into five stages. On the basis of the traditional fuzzy comprehensive evaluation method, the weights were adjusted according to the construction stage, and the risk dynamic evaluation model was finally constructed. The dynamic risk assessment method can be used as a reference for similar tunnels.

\section{Engineering Background}

The ultra-shallow-buried and large-span double-arch tunnel of the land part of the Xiamen Haicang Tunnel is located in Huli District, Xiamen City, Fujian Province, China. The entrance of the double-arch tunnel is at the No. 4 working well on the north slope of Xinghu Road. The exit of the tunnel is at the No. 5 working well near the east exit of the Furongyuan underground parking lot on the south side of Xinghu Road. The starting and ending mileages are BK17 + 825 and BK17 + 982, respectively, and the total length 
is $157 \mathrm{~m}$. The double-arch tunnel underpasses through Xinghu Road, and is a two-way, six-lane urban expressway. The main road of Xinghu Road is crowded with heavy vehicles. Under the road surface, various municipal pipelines, such as water supply, drainage, and power cables, are buried within $2.5 \mathrm{~m}$ below the ground and approximately $1.8 \mathrm{~m}$ to $3.0 \mathrm{~m}$ above the top of the tunnel. Many buildings exist around the tunnel, and the shortest distance from the underground garage of Furongyuan is only approximately $30 \mathrm{~m}$. In the construction range of the underground double-arch tunnel, the surrounding rock is mainly quaternary hybrid fill, silty clay and fully and strongly weathered bedrocks. The roof at the tunnel entrance is in the miscellaneous fill and silty clay layer, and the other main bodies of the tunnel are mainly buried in completely or strongly weathered rocks or residual soil. Figure 1 presents the longitudinal section of the double-arch tunnel. The buried depth of the tunnel is relatively shallow, and the effective pressure arched area cannot form after excavation. Furthermore, the strength of the surrounding rock is low, and its self-stability is poor. Therefore, the stability of the surrounding rock and sidewall of the vault during construction should be a concern. The stability of the rock and soil in the tunnel construction area is poor, and the construction risk is high.

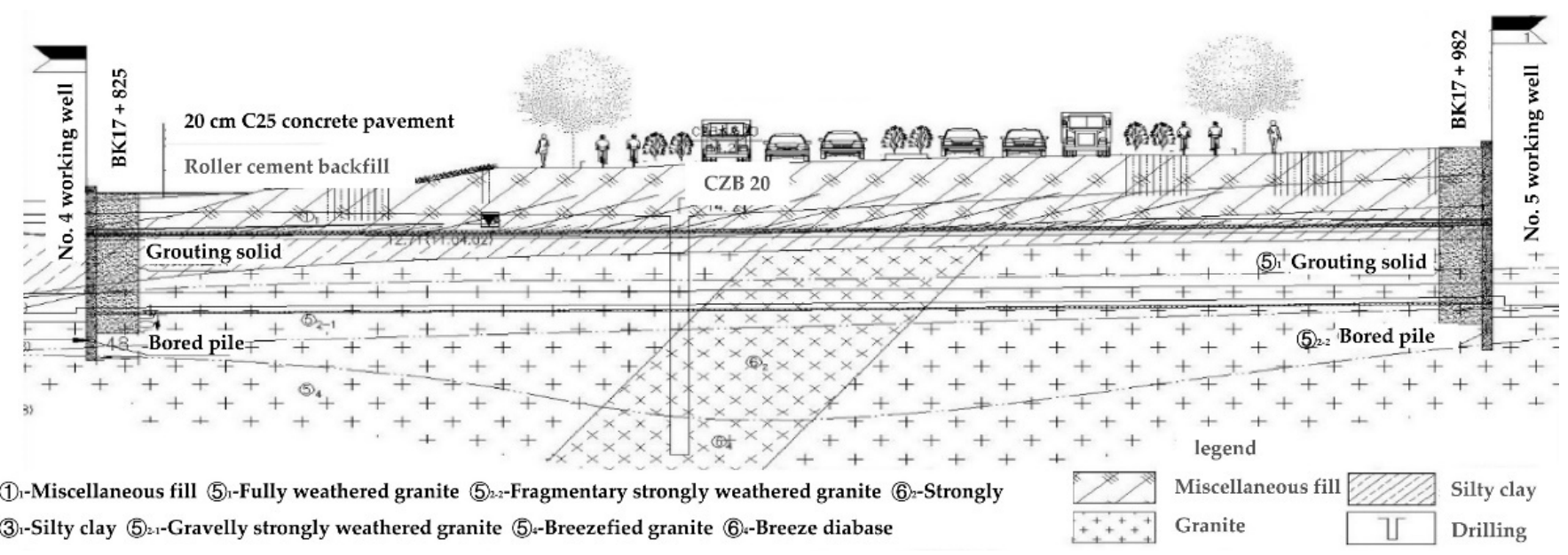

Figure 1. Longitudinal view of the double-arch tunnel.

The tunnel is excavated by using three pilot tunnels and the three-bench reserved core soil method. Before excavation, the ground and the surrounding rock are strengthened. The main construction in this stage includes reinforcements, such as ground grouting reinforcement, grouting reinforcement of the rock surrounding the tunnel, concrete mixing pile reinforcement at the entrance, pipe shed construction at the entrance, and advanced support structure construction, and the excavation of the guide and main tunnels. The sequence during pilot tunnel excavation is as follows: the middle; left; and right pilot tunnels. The spacing between the advanced and following pilot tunnels is $20 \mathrm{~m}$. The partition wall is constructed after the excavation of pilot tunnels. Through the analysis of the above construction methods and procedures, the entire construction process can be divided into five stages. The five stages are as follows: the initial stage/preparation stage before construction; Stage 1, ground and surrounding rock reinforcement stage; Stage 2, pilot tunnel excavation stage; Stage 3, main tunnel excavation stage (single tunnel excavation); Stage 4, main tunnel excavation stage (double tunnel excavation). 


\section{Dynamic Risk Evaluation Index Construction}

The risk assessment process of the construction process of shallow-buried and largespan double-arch tunnel is as follows: (1) The construction risks of urban ultra-shallowburied and large-span double-arch tunnels are identified using two aspects, risk type identification and risk factor identification, and the risk evaluation index suitable for shallow-buried and large-span double-arch tunnel is then proposed; (2) According to the actual working conditions on site and expert opinion, the primary and secondary risk evaluation indexes are weighed up and determined by using the AHP. The weights of risk evaluation indicators are revised according to changes in the monitoring data and construction status; (3) Determine the quantitative value of the membership degree of the construction dynamic risk evaluation index according to expert opinion, and then use the membership degree function to ensure the fuzzy evaluation matrix is composed of these indexes in each stage; (4) According to the weight and membership degree of the second-level risk evaluation index in each construction stage, fuzzy calculation is carried out to obtain the first-level comprehensive evaluation matrix. According to the weight of first-level risk evaluation index and first-level comprehensive evaluation matrix, the second-level comprehensive evaluation matrix is obtained by fuzzy calculation; (5) A quantitative analysis of fuzzy comprehensive evaluation results is conducted.

The establishment of a risk evaluation index system is an important link to risk assessment. The rationality of the index system directly affects the reliability of risk assessment results. The selection of risk evaluation indexes is based on the following criteria: objective; scientific; systematic; comprehensive; easy to quantify; and reflect the research content. The risk evaluation index is determined on the basis of the risk identification results, the risk types, and the risk factors. Risk identification can be divided into risk type identification and risk factor identification. According to the main risk types and risk factors, dynamic construction risk evaluation indexes suitable for urban ultra-shallow large-span double-arch tunnels can be further determined. The construction risk types of urban shallow-buried and concealed double-arch tunnels are divided into tunnel collapse, water and sand penetration, mechanical injury, surrounding environment damage, structural damage, electric shock, fire, explosion, poisoning, and suffocation. According to the key characteristics of the design and construction of urban shallow-buried large-span tunnels [49] and the opinions of tunnel experts, the main risk factors in the construction of urban ultra-shallow buried large-span double-arch tunnels include the engineering geology and hydrogeology, the surrounding construction environment, the overall engineering design, the engineering construction technology, and the engineering construction management. To help risk assessors with indicator weighting, risk classification, and other tasks in the follow-up work, the risk evaluation indexes should be divided into several levels according to a certain logic, which are generally called first-level indexes, secondlevel indexes, and so on. According to existing research $[7,18,49]$ and the opinions of tunnel experts, the first-level index $\left(\mathrm{U}_{\mathrm{i}}\right)$ in the construction risk evaluation system of urban shallow-buried large-span double-arch tunnels was divided into six aspects: engineering geology and hydrogeology; surrounding construction environment; overall engineering design; engineering construction technology; engineering construction management; and monitoring measurement data. Given that monitoring measurement data should be obtained in the construction stage, this indicator is only used during construction, and only the first five indexes need to be used in the preparation stage before construction. Then, the first-level indexes were divided into the corresponding second-level indexes $\left(\mathrm{U}_{\mathrm{ij}}\right)$, which are the $j$-th second-level indexes of the $i$-th first-level index. Finally, an urban shallowburied large-span double-arch tunnel construction dynamic risk evaluation index system composed of six first-level indexes and thirty second-level indexes was established, as shown in Figure 2. 


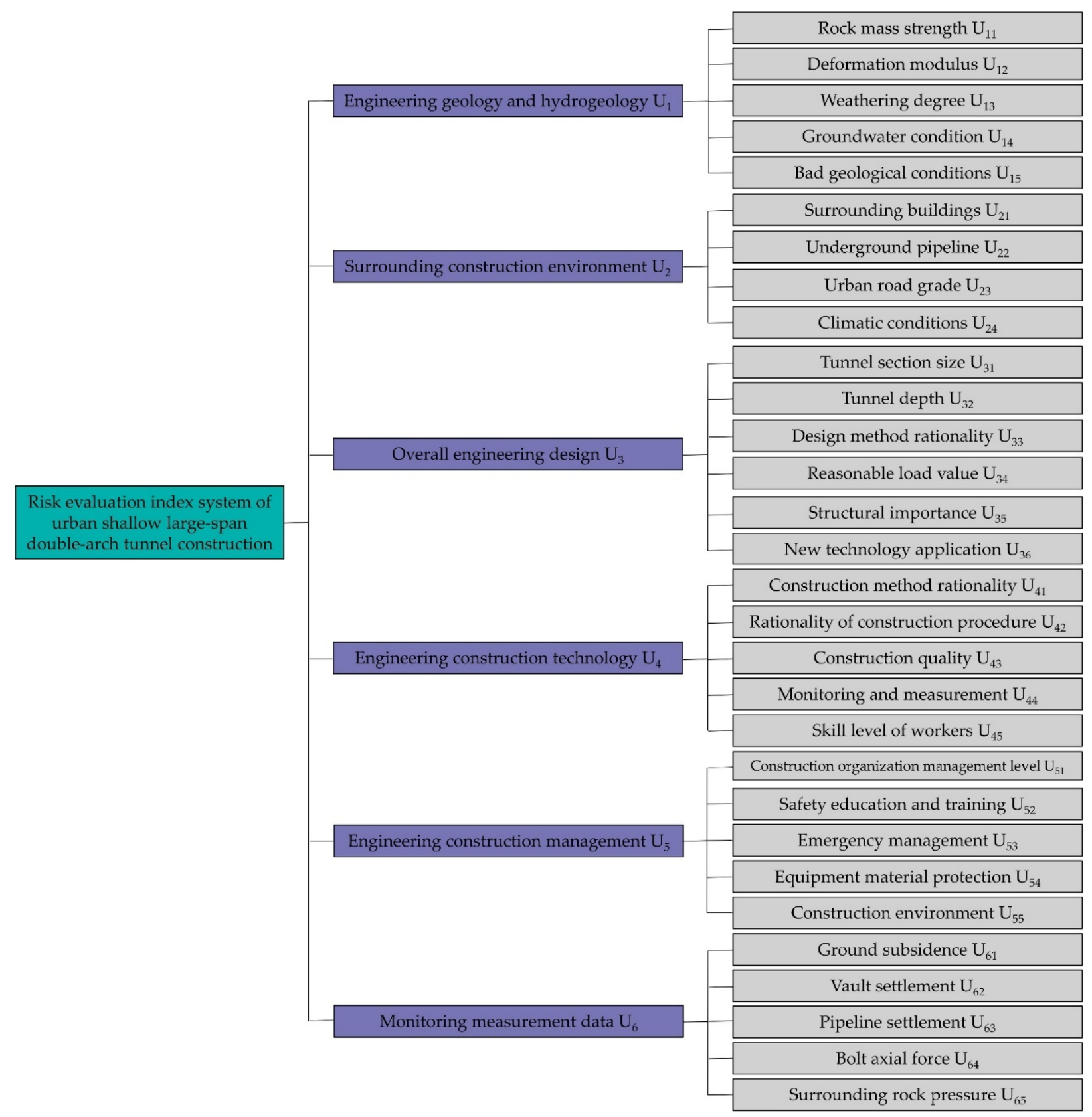

Figure 2. Risk evaluation index system of urban shallow-buried large-span double-arch tunnel construction.

\section{Construction Dynamic Risk Evaluation Index Weight}

\subsection{Benchmark Weight Determination}

According to the existing risk assessment hierarchical system, the commonly used and effective AHP method is used to determine the weight of the indexes. The AHP is a systematic analysis method that combines qualitative and quantitative analyses. The AHP hierarchizes the problem, decomposes it into different components, and combines them in hierarchical clusters according to their mutual influence and affiliation. A judgment matrix is formed by comparing the relative importance of the indexes at each level. The relative importance of each index is quantified using a certain digital scale, which is called the judgment scale, between two indexes. The most commonly used judgment standard would use the numbers 1-9 to represent the relative importance of the indexes, that is, one represents equal importance, and nine represents extreme importance [50]. The degree of importance increases with the digital value, and the criteria are listed in Table 1. 
Table 1. Relative comparison scale.

\begin{tabular}{cc}
\hline Digital Scale & Definition \\
\hline 1 & $U_{\mathrm{i}}$ is as important as $\mathrm{U}_{\mathrm{j}}$ \\
3 & $\mathrm{U}_{\mathrm{i}}$ is slightly more important than $\mathrm{U}_{\mathrm{j}}$ \\
5 & $\mathrm{U}_{\mathrm{i}}$ is obviously more important than $\mathrm{U}_{\mathrm{j}}$ \\
9 & $\mathrm{U}_{\mathrm{i}}$ is more important than $\mathrm{U}_{\mathrm{j}}$ \\
$2,4,6,8$ & $\mathrm{U}_{\mathrm{i}}$ is extremely important than $\mathrm{U}_{\mathrm{j}}$ \\
\hline
\end{tabular}

The importance of each evaluation index at the same level is compared in pairs in order to construct a judgment matrix. Assuming $n$ factors $U_{1}, U_{2}, \ldots, U_{n}$ in a level, scale $a_{i j}$ with a value of 1-9 is used to reflect the relative importance of the comparison between $U_{i}$ and $U_{j}$, and an $n$-th order judgment matrix is obtained, as shown in Equation (1).

$$
A=\left[\begin{array}{ccc}
a_{11} & \cdots & a_{1 n} \\
\vdots & & \vdots \\
a_{n 1} & \cdots & a_{n n}
\end{array}\right]
$$

The maximum eigenvalue $\left(\lambda_{\max }\right)$ and eigenvector $(W)$ of the judgment matrix are obtained, and the eigenvector is normalized to obtain the relative weight of the risk indexes at the same level. Then, the consistency test is carried out to determine the logical rationality of the judgment matrix. The random consistency ratio $\left(C_{R}\right)$ is used to check the consistency of the judgment matrix. When $C_{R}$ is less than 0.1 , the requirement is met.

$$
C_{R}=\frac{C_{I}}{R_{I}}
$$

where $R_{I}$ is the average random consistency index value of the judgment matrix, as shown in Table 2. $C_{I}$ is the consistency index, which can be calculated by Equation (3).

$$
C_{I}=\frac{\lambda_{\max }-n}{n-1}
$$

Table 2. Average random consistency index $R_{I}$.

\begin{tabular}{cccccccccc}
\hline Matrix Order & $\mathbf{2}$ & $\mathbf{3}$ & $\mathbf{4}$ & $\mathbf{5}$ & $\mathbf{6}$ & $\mathbf{7}$ & $\mathbf{8}$ & $\mathbf{9}$ & $\mathbf{1 0}$ \\
\hline$R_{I}$ & 0 & 0.58 & 0.90 & 1.12 & 1.24 & 1.32 & 1.41 & 1.46 & 1.49 \\
\hline
\end{tabular}

Ten experienced tunnel design, geological exploration, and tunnel construction experts were selected in order to form an evaluation team. Through the table comparison method [51], the experts scored the importance of each level of risk factors. According to the experts' replies, a judgment matrix was constructed. According to the AHP method, the relative weights of the first- and second-level risk evaluation indexes were calculated, and the results are shown in Tables 3 and 4. 
Table 3. Weight calculation of first-level risk evaluation indexes.

\begin{tabular}{|c|c|c|c|c|c|c|c|c|c|c|}
\hline \multirow{2}{*}{ Stage } & \multirow{2}{*}{ First-Level Indexes } & \multicolumn{6}{|c|}{ First-Level Indexes } & \multirow{2}{*}{$C_{I}$} & \multirow{2}{*}{$C_{R}$} & \multirow{2}{*}{ Weight } \\
\hline & & $\mathrm{U}_{1}$ & $\mathrm{U}_{2}$ & $\mathrm{U}_{3}$ & $\mathrm{U}_{4}$ & $\mathrm{U}_{5}$ & $\mathrm{U}_{6}$ & & & \\
\hline \multirow{5}{*}{$\begin{array}{l}\text { Preparation } \\
\text { stage before } \\
\text { construction }\end{array}$} & $\begin{array}{c}\text { Engineering geology and } \\
\text { hydrogeology } U_{1}\end{array}$ & 1 & 2 & 1 & 2 & 5 & & \multirow{5}{*}{0.0135} & \multirow{5}{*}{0.0121} & 0.32 \\
\hline & $\begin{array}{l}\text { Surrounding construction } \\
\text { environment } U_{2}\end{array}$ & $\frac{1}{2}$ & 1 & $1 / 2$ & 1 & 2 & & & & 0.15 \\
\hline & Overall engineering design $U_{3}$ & 1 & 2 & 1 & 1 & 4 & & & & 0.27 \\
\hline & $\begin{array}{l}\text { Engineering construction } \\
\text { technology } U_{4}\end{array}$ & $\frac{1}{2}$ & 1 & 1 & 1 & 3 & & & & 0.19 \\
\hline & $\begin{array}{l}\text { Engineering construction } \\
\text { management } U_{5}\end{array}$ & $1 / 5$ & $1 / 2$ & $1 / 4$ & $1 / 3$ & 1 & & & & 0.07 \\
\hline \multirow{6}{*}{$\begin{array}{l}\text { Construction } \\
\text { stage }\end{array}$} & $\begin{array}{c}\text { Engineering geology and } \\
\text { hydrogeology } U_{1}\end{array}$ & 1 & 2 & 1 & 2 & 5 & 1 & \multirow{6}{*}{0.011} & \multirow{6}{*}{0.0089} & 0.24 \\
\hline & $\begin{array}{l}\text { Surrounding construction } \\
\text { environment } U_{2}\end{array}$ & $\frac{1}{2}$ & 1 & $1 / 2$ & 1 & 2 & $1 / 2$ & & & 0.12 \\
\hline & Overall engineering design $\mathrm{U}_{3}$ & 1 & 2 & 1 & 1 & 4 & 1 & & & 0.21 \\
\hline & $\begin{array}{l}\text { Engineering construction } \\
\text { technology } U_{4}\end{array}$ & $\frac{1}{2}$ & 1 & 1 & 1 & 3 & $1 / 2$ & & & 0.14 \\
\hline & $\begin{array}{l}\text { Engineering construction } \\
\text { management } U_{5}\end{array}$ & $1 / 5$ & $1 / 2$ & $1 / 4$ & $1 / 3$ & 1 & $1 / 5$ & & & 0.05 \\
\hline & Monitoring measurement data $\mathrm{U}_{6}$ & 1 & 2 & 1 & 2 & 5 & 1 & & & 0.24 \\
\hline
\end{tabular}

Table 4. Weight of second-level evaluation indexes.

\begin{tabular}{|c|c|c|c|c|c|c|c|c|c|c|}
\hline \multirow{2}{*}{$\begin{array}{l}\text { First-Level } \\
\text { Indexes }\end{array}$} & \multirow{2}{*}{ Second-Level Indexes } & \multicolumn{6}{|c|}{ Second-Level Indexes } & \multirow{2}{*}{$C_{I}$} & \multirow{2}{*}{$C_{R}$} & \multirow{2}{*}{ Weight } \\
\hline & & $\mathrm{U}_{\mathrm{i} 1}$ & $\mathrm{U}_{\mathrm{i} 2}$ & $\mathrm{U}_{\mathrm{i} 3}$ & $\mathrm{U}_{\mathrm{i} 4}$ & $\mathrm{U}_{\mathrm{i} 5}$ & $\mathrm{U}_{\mathrm{i} 6}$ & & & \\
\hline \multirow{5}{*}{$\begin{array}{l}\text { Engineering } \\
\text { geology and } \\
\text { hydrogeol- } \\
\text { ogy U } U_{1}\end{array}$} & Rock mass strength $\mathrm{U}_{11}$ & 1 & 2 & 5 & 4 & 2 & & \multirow{5}{*}{0.0045} & \multirow{5}{*}{0.004} & 0.40 \\
\hline & Deformation modulus $U_{12}$ & $\frac{1}{2}$ & 1 & 3 & 2 & 1 & & & & 0.21 \\
\hline & Weathering degree $U_{13}$ & $1 / 5$ & $1 / 3$ & 1 & 1 & $1 / 3$ & & & & 0.08 \\
\hline & Groundwater condition $U_{14}$ & $\frac{1}{4}$ & $1 / 2$ & 1 & 1 & $1 / 2$ & & & & 0.10 \\
\hline & Bad geological conditions $U_{15}$ & $\frac{4}{2}$ & 1 & 3 & 2 & 1 & & & & 0.21 \\
\hline \multirow{4}{*}{$\begin{array}{l}\text { Surrounding } \\
\text { construction } \\
\text { environ- } \\
\text { ment } U_{2}\end{array}$} & Surrounding buildings $U_{21}$ & 1 & 2 & 3 & 4 & & & \multirow{4}{*}{0.0103} & \multirow{4}{*}{0.0115} & 0.47 \\
\hline & Underground pipeline $U_{22}$ & $\frac{1}{2}$ & 1 & 2 & 3 & & & & & 0.28 \\
\hline & Urban road grade $U_{23}$ & $17^{2} 3$ & $1 / 2$ & 1 & 1 & & & & & 0.14 \\
\hline & Climatic conditions $\mathrm{U}_{24}$ & $\frac{1}{4}$ & $1 / 3$ & 1 & 1 & & & & & 0.11 \\
\hline \multirow{6}{*}{$\begin{array}{c}\text { Overall } \\
\text { engineering } \\
\text { design } U_{3}\end{array}$} & Tunnel section size $U_{31}$ & 1 & 2 & 1 & 1 & 3 & 3 & \multirow{6}{*}{0.0109} & \multirow{6}{*}{0.0088} & 0.24 \\
\hline & Tunnel depth $\mathrm{U}_{32}$ & $\frac{1}{2}$ & 1 & $\frac{1}{2}$ & $1 / 2$ & 2 & 2 & & & 0.13 \\
\hline & Design method rationality $\mathrm{U}_{33}$ & $\stackrel{2}{1}$ & 2 & $\stackrel{2}{1}$ & 1 & 2 & 3 & & & 0.23 \\
\hline & Reasonable load value $U_{34}$ & 1 & 2 & 1 & 1 & 2 & 3 & & & 0.23 \\
\hline & Structural importance $U_{35}$ & $1 / 3$ & $1 / 2$ & $\frac{1}{2}$ & $1 / 2$ & 1 & 1 & & & 0.09 \\
\hline & New technology application $U_{36}$ & $1 / 3$ & $1 / 2$ & 173 & $1 / 3$ & 1 & 1 & & & 0.08 \\
\hline \multirow{5}{*}{$\begin{array}{l}\text { Engineering } \\
\text { construction } \\
\text { technology } U_{4}\end{array}$} & Construction method rationality $\mathrm{U}_{41}$ & 1 & 2 & $1 / 3$ & 2 & 2 & & \multirow{5}{*}{0.0046} & \multirow{5}{*}{0.0041} & 0.20 \\
\hline & Rationality of construction procedure $U_{42}$ & $\frac{1}{2}$ & 1 & $\frac{1}{4}$ & 1 & 1 & & & & 0.11 \\
\hline & Construction quality $\mathrm{U}_{43}$ & $\overline{2}$ & 4 & $\frac{4}{1}$ & 4 & 5 & & & & 0.48 \\
\hline & Monitoring and measurement $\mathrm{U}_{44}$ & $\frac{1}{2}$ & 1 & $\frac{1}{4}$ & 1 & 1 & & & & 0.11 \\
\hline & Skill level of workers $U_{45}$ & $\frac{1}{2}$ & 1 & $1 / 5$ & 1 & 1 & & & & 0.10 \\
\hline \multirow{5}{*}{$\begin{array}{c}\text { Engineering } \\
\text { construction } \\
\text { manage- } \\
\text { ment } U_{5}\end{array}$} & $\begin{array}{c}\text { Construction organization management } \\
\text { level } U_{51}\end{array}$ & 1 & $1 / 2$ & 2 & 2 & 3 & & \multirow{5}{*}{0.0123} & \multirow{5}{*}{0.0110} & 0.24 \\
\hline & Safety education and training $U_{52}$ & 2 & 1 & 3 & 4 & 4 & & & & 0.42 \\
\hline & Emergency management $\mathrm{U}_{53}$ & $\frac{1}{2}$ & $1 / 3$ & 1 & 1 & 2 & & & & 0.14 \\
\hline & Equipment material protection $\mathrm{U}_{54}$ & $\frac{1}{2}$ & $1 / 4$ & 1 & 1 & 1 & & & & 0.11 \\
\hline & Construction environment $\mathrm{U}_{55}$ & $1 / 3$ & $1 / 4$ & $\frac{1}{2}$ & 1 & 1 & & & & 0.09 \\
\hline \multirow{5}{*}{$\begin{array}{l}\text { Monitoring } \\
\text { measurement } \\
\text { data } \mathrm{U}_{6}\end{array}$} & Ground subsidence $\mathrm{U}_{61}$ & 1 & $1 / 2$ & 1 & 3 & 4 & & \multirow{5}{*}{0.0096} & \multirow{5}{*}{0.0086} & 0.24 \\
\hline & Vault settlement $U_{62}$ & 2 & 1 & 2 & 4 & 5 & & & & 0.40 \\
\hline & Pipeline settlement $U_{63}$ & 1 & $1 / 2$ & 1 & 2 & 3 & & & & 0.20 \\
\hline & Bolt axial force $U_{64}$ & $1 / 3$ & $1 / 4$ & $\frac{1}{2}$ & 1 & 1 & & & & 0.09 \\
\hline & Surrounding rock pressure $\mathrm{U}_{65}$ & $\frac{1}{4}$ & $1 / 5$ & $1 / 3$ & 1 & 1 & & & & 0.07 \\
\hline
\end{tabular}




\subsection{Base Weight Correction}

During construction, the pairwise judgment relationship of the risk evaluation indexes at the same level may change. To ensure the accuracy of the construction risk assessment results, the benchmark weights should be corrected according to the construction stage. According to reference [49] and relevant specifications and expert opinions, the construction stage of each risk assessment index was quantified. The classification standard for the change in construction stage of each index can be based on the reference value of the risk evaluation index classification standard, as shown in Table 5.

Table 5. The reference value of risk evaluation index classification standard.

\begin{tabular}{|c|c|c|c|c|c|}
\hline \multirow{2}{*}{ Second-Level Indexes } & \multicolumn{5}{|c|}{ Risk Level and Scoring Range } \\
\hline & I (90 100) & II (80 90) & III (70 80) & IV (60 70) & $\mathrm{V}(0 \sim 60)$ \\
\hline $\begin{array}{l}\text { Rock mass } \\
\text { strength } U_{11}\end{array}$ & $>60$ & $30 \sim 60$ & $15 \sim 30$ & $5 \sim 15$ & $<5$ \\
\hline $\begin{array}{l}\text { Deformation } \\
\text { modulus } U_{12}\end{array}$ & $>50$ & $30 \sim 50$ & $10 \sim 30$ & $5 \sim 10$ & $<5$ \\
\hline $\begin{array}{l}\text { Weathering } \\
\text { degree } U_{13}\end{array}$ & Unweathered & Breeze & Moderately weathered & Strong weathered & Completely weathered \\
\hline $\begin{array}{l}\text { Groundwater } \\
\text { condition } U_{14}\end{array}$ & No water & Less water & Moderately water & More water & Abundant water \\
\hline $\begin{array}{l}\text { Bad geological } \\
\text { conditions } U_{15}\end{array}$ & No bad geology & Less bad geology & Poor geology exists & More bad geology & $\begin{array}{l}\text { Abundant bad } \\
\text { geology }\end{array}$ \\
\hline $\begin{array}{l}\text { Surrounding } \\
\text { buildings } U_{21}\end{array}$ & $\begin{array}{l}\text { No surrounding } \\
\text { buildings }\end{array}$ & Secondary buildings & $\begin{array}{l}\text { Buildings of general } \\
\text { importance }\end{array}$ & Important building & $\begin{array}{l}\text { Key protected } \\
\text { buildings }\end{array}$ \\
\hline $\begin{array}{l}\text { Underground } \\
\text { pipeline } U_{22}\end{array}$ & No pipeline & Less pipeline & More pipelines & Dense pipeline & $\begin{array}{l}\text { Pipeline must be } \\
\text { relocated }\end{array}$ \\
\hline Urban road grade $\mathrm{U}_{23}$ & Branch road & Secondary trunk road & Trunk road & Expressway & $\begin{array}{l}\text { Multiple roads and } \\
\text { complex routes }\end{array}$ \\
\hline $\begin{array}{c}\text { Climatic } \\
\text { conditions } U_{24}\end{array}$ & Good & Better & Generally & Poor & Very bad \\
\hline $\begin{array}{l}\text { Tunnel section } \\
\text { size } U_{31}\end{array}$ & $<10$ & $10 \sim 30$ & $30 \sim 50$ & $50 \sim 100$ & $>100$ \\
\hline Tunnel depth $\mathrm{U}_{32}$ & $>30$ & $22 \sim 30$ & $15 \sim 22$ & $8 \sim 15$ & $<8$ \\
\hline $\begin{array}{l}\text { Design method } \\
\text { rationality } \mathrm{U}_{33}\end{array}$ & Reasonable & Basically reasonable & General reasonable & Poor & Unreasonable \\
\hline $\begin{array}{l}\text { Reasonable load } \\
\text { value } U_{34}\end{array}$ & Reasonable & Basically reasonable & General reasonable & Poor & Unreasonable \\
\hline $\begin{array}{c}\text { Structural } \\
\text { importance } U_{35}\end{array}$ & Unimportant & Basically important & General important & More important & Very important \\
\hline $\begin{array}{l}\text { New technology } \\
\text { application } \mathrm{U}_{36}\end{array}$ & No new technology & Few new technologies & $\begin{array}{l}\text { General new } \\
\text { technologies }\end{array}$ & $\begin{array}{l}\text { More new } \\
\text { technologies }\end{array}$ & $\begin{array}{l}\text { Many new } \\
\text { technologies }\end{array}$ \\
\hline $\begin{array}{l}\text { Construction method } \\
\text { rationality } \mathrm{U}_{41}\end{array}$ & Reasonable & Basically reasonable & General reasonable & Poor & Unreasonable \\
\hline $\begin{array}{l}\text { Rationality of } \\
\text { construction } \\
\text { procedure } U_{42}\end{array}$ & Reasonable & Basically reasonable & General reasonable & Poor & Unreasonable \\
\hline $\begin{array}{l}\text { Construction } \\
\text { quality } \mathrm{U}_{43}\end{array}$ & $\begin{array}{l}\text { Superior to design } \\
\text { standards }\end{array}$ & Meet design standards & General quality & Below quality & $\begin{array}{l}\text { Seriously unqualified } \\
\text { quality }\end{array}$ \\
\hline $\begin{array}{l}\text { Monitoring and } \\
\text { measurement } U_{44}\end{array}$ & $\begin{array}{l}\text { Strictly follow the } \\
\text { specifications }\end{array}$ & More perfection & General perfection & Imperfect & $\begin{array}{l}\text { None Monitored } \\
\text { measurement }\end{array}$ \\
\hline 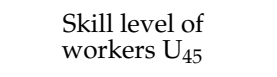 & $\begin{array}{l}\text { Perfect arrangement } \\
\text { of technical staff }\end{array}$ & $\begin{array}{l}\text { The arrangement of } \\
\text { technical staff is } \\
\text { relatively complete }\end{array}$ & $\begin{array}{l}\text { Equipped with basic } \\
\text { technical staff }\end{array}$ & $\begin{array}{c}\text { Lack of key technical } \\
\text { staff }\end{array}$ & $\begin{array}{l}\text { Severe shortage of } \\
\text { technical staff }\end{array}$ \\
\hline $\begin{array}{c}\text { Construction } \\
\text { organization } \\
\text { management level } \mathrm{U}_{51}\end{array}$ & No omissions & $\begin{array}{c}\text { Can better complete } \\
\text { the construction } \\
\text { content }\end{array}$ & $\begin{array}{l}\text { Can basically } \\
\text { complete the } \\
\text { construction content }\end{array}$ & Poor & Very bad \\
\hline $\begin{array}{l}\text { Safety education and } \\
\text { training } U_{52}\end{array}$ & Adequate training & More training & General training & Less training & No training \\
\hline $\begin{array}{c}\text { Emergency } \\
\text { management } U_{53}\end{array}$ & $\begin{array}{l}\text { Perfect emergency } \\
\text { plan }\end{array}$ & Relatively complete & General perfection & $\begin{array}{l}\text { Incomplete emergency } \\
\text { plan }\end{array}$ & No contingency plan \\
\hline $\begin{array}{l}\text { Equipment material } \\
\text { protection } U_{54}\end{array}$ & New equipment & $\begin{array}{c}\text { Relatively new } \\
\text { equipment }\end{array}$ & $\begin{array}{l}\text { New equipment but } \\
\text { poor maintenance }\end{array}$ & $\begin{array}{l}\text { Equipment is new and } \\
\text { maintenance is not } \\
\text { timely }\end{array}$ & $\begin{array}{l}\text { Serious aging of } \\
\text { equipment }\end{array}$ \\
\hline $\begin{array}{l}\text { Construction } \\
\text { environment } U_{55}\end{array}$ & $\begin{array}{c}\text { No impact on } \\
\text { construction safety }\end{array}$ & Relatively good & Generally & Poor & Very bad \\
\hline $\begin{array}{c}\text { Ground } \\
\text { subsidence } \mathrm{U}_{61}\end{array}$ & $\mathrm{~S} 1 \leq 0.6 \mathrm{U}_{1}$ & $0.6 \mathrm{U}_{1}<\mathrm{S} 1 \leq 0.7 \mathrm{U}_{1}$ & $0.7 \mathrm{U}_{1}<\mathrm{S} 1 \leq 0.8 \mathrm{U}_{1}$ & $0.8 \mathrm{U}_{1}<\mathrm{S} 1 \leq 0.9 \mathrm{U}_{1}$ & $\mathrm{~S} 1 \geq 0.9 \mathrm{U}_{1}$ \\
\hline Vault settlement $U_{62}$ & $\mathrm{~S} 2 \leq 0.6 \mathrm{U}_{2}$ & $0.6 \mathrm{U}<2 \mathrm{~S} 2 \leq 0.7 \mathrm{U}_{2}$ & $0.7 \mathrm{U}_{2}<\mathrm{S} 2 \leq 0.8 \mathrm{U}_{2}$ & $0.8 \mathrm{U}_{2}<\mathrm{S} 2 \leq 0.9 \mathrm{U}_{2}$ & $\mathrm{~S} 2 \geq 0.9 \mathrm{U}_{2}$ \\
\hline $\begin{array}{l}\text { Pipeline } \\
\text { settlement } U_{63}\end{array}$ & $\mathrm{~S} 3 \leq 0.6 \mathrm{U}_{3}$ & $0.6 \mathrm{U}<3 \mathrm{~S} 3 \leq 0.7 \mathrm{U}_{3}$ & $0.7 \mathrm{U}_{3}<\mathrm{S} 3 \leq 0.8 \mathrm{U}_{3}$ & $0.8 \mathrm{U}_{3}<\mathrm{S} 3 \leq 0.9 \mathrm{U}_{3}$ & $\mathrm{~S} 3 \geq 0.9 \mathrm{U}_{3}$ \\
\hline Bolt axial force $\mathrm{U}_{64}$ & $n \leq 0.6 \mathrm{U}_{4}$ & $0.6 \mathrm{U}_{4}<n \leq 0.7 \mathrm{U}_{4}$ & $0.7 \mathrm{U}_{4}<n \leq 0.8 \mathrm{U}_{4}$ & $0.8 \mathrm{U}_{4}<n \leq 0.9 \mathrm{U}_{4}$ & $n \geq 0.9 \mathrm{U}_{4}$ \\
\hline $\begin{array}{l}\text { Surrounding rock } \\
\text { pressure } U_{65}\end{array}$ & $p \leq 0.6 \mathrm{U}_{5}$ & $0.6 \mathrm{U}_{5}<p \leq 0.7 \mathrm{U}_{5}$ & $0.7 \mathrm{U}_{5}<p \leq 0.8 \mathrm{U}_{5}$ & $0.8 \mathrm{U}_{5}<p \leq 0.9 \mathrm{U}_{5}$ & $p \geq 0.9 \mathrm{U}_{5}$ \\
\hline
\end{tabular}

Note: S1, S2, and S3, respectively, represent the monitoring values of the ground, vault, and pipeline settlements. $U_{1}, U_{2}$, and $U_{3}$, respectively, represent the control values of the ground, vault, and pipeline settlements. $n$ and $p$, respectively, represent the monitoring values of the bolt axial force and the surrounding rock pressure. U4 and U5, respectively, represent the allowable values of bolt axial force and surrounding rock pressure. 
After scoring the risk evaluation index according to the construction state, a vector was obtained and normalized to obtain the weight correction vector $\mathrm{X}\left(x_{1}, x_{2}, \ldots, x_{\mathrm{i}}\right) . x_{\mathrm{i}}$ is the normalized value of the construction state of the risk evaluation index. The benchmark weight can be modified according to Equation (4).

$$
Y_{\mathrm{i}}(X)=\frac{y_{\mathrm{i}} x_{\mathrm{i}}^{-\frac{1}{2}}}{\sum_{\mathrm{i}=1}^{m} y_{\mathrm{i}} x_{\mathrm{i}}^{-\frac{1}{2}}}
$$

where $Y_{i}(X)$ is the weight correction value of each risk assessment index, and $y_{\mathrm{i}}$ is the initial weight value.

According to monitoring data and the changes in the construction at each stage, the benchmark weights of the risk assessment indicators were adjusted, as shown in Table 6 .

Table 6. The dynamic weight of each stage.

\begin{tabular}{|c|c|c|c|c|c|}
\hline \multirow{2}{*}{ Second-Level Indexes } & \multicolumn{5}{|c|}{ The Dynamic Weight of Each Stage } \\
\hline & Initial Stage & Stage 1 & Stage 2 & Stage 3 & Stage 4 \\
\hline Rock mass strength $U_{11}$ & 0.40 & 0.38 & 0.30 & 0.36 & 0.24 \\
\hline Deformation modulus $U_{12}$ & 0.21 & 0.23 & 0.19 & 0.24 & 0.16 \\
\hline Weathering degree $U_{13}$ & 0.08 & 0.10 & 0.12 & 0.12 & 0.12 \\
\hline Groundwater condition $U_{14}$ & 0.10 & 0.08 & 0.09 & 0.17 & 0.08 \\
\hline Bad geological conditions $U_{15}$ & 0.21 & 0.21 & 0.30 & 0.11 & 0.40 \\
\hline Surrounding buildings $\mathrm{U}_{21}$ & 0.47 & 0.38 & 0.35 & 0.40 & 0.36 \\
\hline Underground pipeline $U_{22}$ & 0.28 & 0.26 & 0.28 & 0.27 & 0.26 \\
\hline Urban road grade $U_{23}$ & 0.14 & 0.21 & 0.23 & 0.20 & 0.18 \\
\hline Climatic conditions $\mathrm{U}_{24}$ & 0.11 & 0.15 & 0.14 & 0.13 & 0.20 \\
\hline Tunnel section size $\mathrm{U}_{31}$ & 0.24 & 0.25 & 0.25 & 0.21 & 0.38 \\
\hline Tunnel depth $\mathrm{U}_{32}$ & 0.13 & 0.16 & 0.18 & 0.25 & 0.26 \\
\hline Design method rationality $U_{33}$ & 0.23 & 0.20 & 0.19 & 0.17 & 0.10 \\
\hline Reasonable load value $U_{34}$ & 0.23 & 0.18 & 0.18 & 0.17 & 0.10 \\
\hline Structural importance $\mathrm{U}_{35}$ & 0.09 & 0.09 & 0.08 & 0.10 & 0.08 \\
\hline New technology application $U_{36}$ & 0.08 & 0.12 & 0.12 & 0.10 & 0.08 \\
\hline Construction method rationality $\mathrm{U}_{41}$ & 0.20 & 0.20 & 0.21 & 0.22 & 0.22 \\
\hline Rationality of construction procedure $\mathrm{U}_{42}$ & 0.11 & 0.11 & 0.15 & 0.16 & 0.16 \\
\hline Construction quality $\mathrm{U}_{43}$ & 0.48 & 0.46 & 0.38 & 0.36 & 0.38 \\
\hline Monitoring and measurement $\mathrm{U}_{44}$ & 0.11 & 0.11 & 0.12 & 0.12 & 0.11 \\
\hline Skill level of workers $U_{45}$ & 0.10 & 0.12 & 0.14 & 0.14 & 0.13 \\
\hline Construction organization management level $U_{51}$ & 0.24 & 0.25 & 0.24 & 0.24 & 0.24 \\
\hline Safety education and training $U_{52}$ & 0.42 & 0.35 & 0.38 & 0.38 & 0.39 \\
\hline Emergency management $\mathrm{U}_{53}$ & 0.14 & 0.16 & 0.16 & 0.15 & 0.15 \\
\hline Equipment material protection $\mathrm{U}_{54}$ & 0.11 & 0.12 & 0.10 & 0.12 & 0.12 \\
\hline Construction environment $\mathrm{U}_{55}$ & 0.09 & 0.12 & 0.12 & 0.11 & 0.10 \\
\hline Ground subsidence $\mathrm{U}_{61}$ & & 0.50 & 0.25 & 0.24 & 0.25 \\
\hline Vault settlement $U_{62}$ & & 0.00 & 0.36 & 0.31 & 0.31 \\
\hline Pipeline settlement $U_{63}$ & & 0.50 & 0.18 & 0.20 & 0.19 \\
\hline Bolt axial force $\mathrm{U}_{64}$ & & 0.00 & 0.12 & 0.15 & 0.15 \\
\hline Surrounding rock pressure $\mathrm{U}_{65}$ & & 0.00 & 0.09 & 0.10 & 0.10 \\
\hline
\end{tabular}

\section{Fuzzy Comprehensive Assessment}

The fuzzy comprehensive assessment method is a comprehensive assessment method based on fuzzy mathematics. The basic principle of the comprehensive assessment method is to quantitatively analyze and comprehensively evaluate the risk factors that have unclear boundaries and are difficult to quantify according to the membership theory of fuzzy mathematics; that is, using fuzzy mathematics to make an overall assessment of the things or objects restricted by many factors. Using this method, the assessment set of a group of risk evaluation indexes was determined first. Then, the weight of each index and their membership degree were determined to obtain the fuzzy evaluation matrix. Finally, the 
weight vector and the fuzzy evaluation matrix were used in the fuzzy operation and normalization processing to obtain the fuzzy comprehensive assessment results.

\subsection{Determine Comment Set}

According to the principle of the fuzzy comprehensive assessment method, the comment set of the risk evaluation index must be determined before calculating the membership degree. The risk evaluation index comment set is a description of the risk grade and severity and a form of risk quantification. According to the actual construction risks of urban shallow-buried large-span double-arch tunnels, the risk assessment indexes can be divided into five levels: safe; relatively safe; generally safe; relatively dangerous; and dangerous. Figure 3 shows the risk comment level and the corresponding quantitative interval of the urban shallow-buried large-span double-arch tunnel construction process.

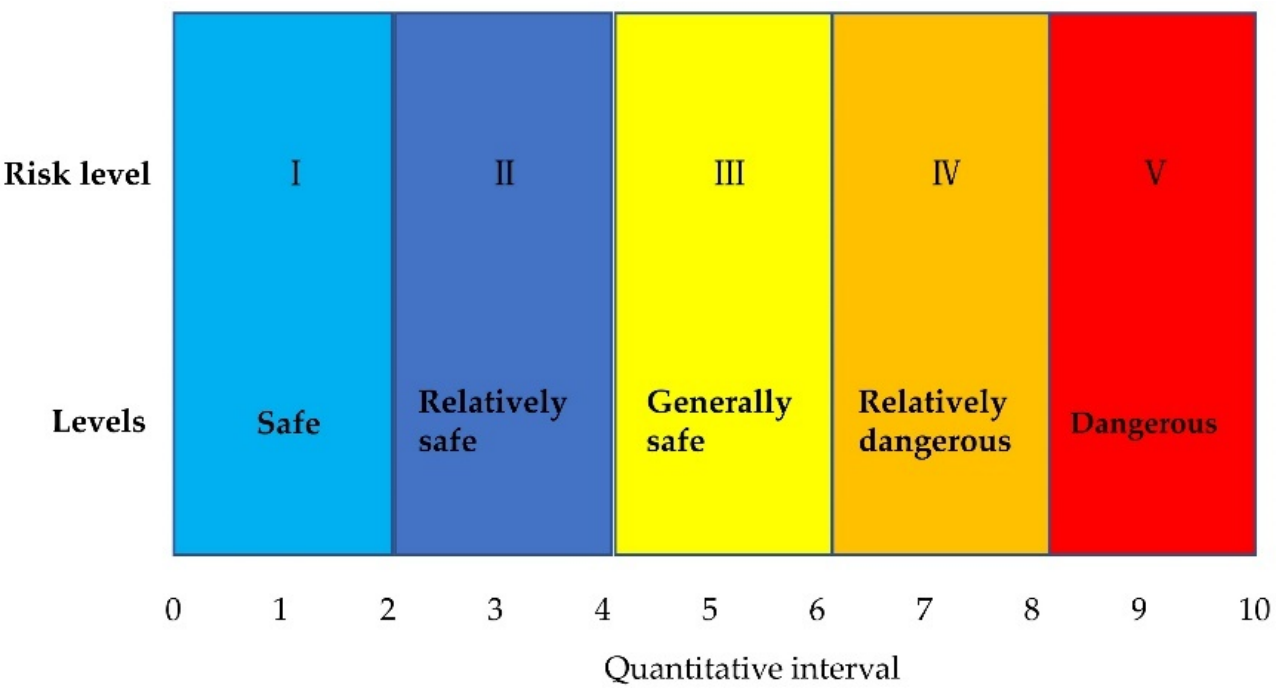

Figure 3. Risk comment level and the corresponding quantitative interval.

\subsection{Determine the Fuzzy Evaluation Matrix}

Referring to the research results of other experts and scholars [49], the trapezoidal function was used to determine the membership degree in the urban shallow-buried largespan double-arch tunnel. Equations (5)-(9) are the membership function relationships of the method.

$$
\begin{aligned}
& u_{1}(x)=\left\{\begin{array}{ll}
0 & x \geq 2.5 \\
2.5-x & 1.5 \leq x \leq 2.5 \\
1 & x<1.5
\end{array},\right. \\
& u_{2}(x)= \begin{cases}0 & x<1.5 \\
x-1.5 & 1.5 \leq x<2.5 \\
1 & 2.5 \leq x<3.5 \\
4.5-x & 3.5 \leq x<4.5 \\
0 & x \geq 4.5\end{cases} \\
& u_{3}(x)= \begin{cases}0 & x<3.5 \\
x-3.5 & 3.5 \leq x<4.5 \\
1 & 4.5 \leq x<5.5 \\
6.5-x & 5.5 \leq x<6.5 \\
0 & x \geq 6.5\end{cases}
\end{aligned}
$$




$$
\begin{aligned}
& u_{4}(x)= \begin{cases}0 & x<5.5 \\
x-5.5 & 5.5 \leq x<6.5 \\
1 & 6.5 \leq x<7.5 \\
8.5-x & 7.5 \leq x<8.5 \\
0 & x \geq 8.5\end{cases} \\
& u_{5}(x)= \begin{cases}1 & x \geq 8.5 \\
x-7.5 & 7.5 \leq x<8.5 \\
0 & x \leq 7.5\end{cases}
\end{aligned}
$$

According to the risk assessment index classification standard and quantification interval and the actual situation of each construction stage of the project, the expert survey method was used to evaluate the quantitative value of the membership degree of the risk evaluation indexes in the pre-construction preparation stage and each construction stage.

The quantitative value of the membership degree of the risk evaluation indexes obtained according to the experts' replies are shown in Figure 4.

The obtained standard of risk assessment index membership degree was substituted into the trapezoidal membership function formula for calculation, and the fuzzy evaluation matrix composed of these indexes was obtained at each construction stage, as shown in Table 7.

\subsection{Results and Analysis of Fuzzy Comprehensive Assessment}

The dynamic risk evaluation model for the construction of the urban shallow-buried large-span double-arch tunnel can be expressed by Equation (10):

$$
[B]_{t}=[w]_{t} \circ\left[\begin{array}{c}
B_{1} \\
B_{2} \\
\vdots \\
B_{5}
\end{array}\right]=[w]_{t} \circ\left\{\left[\begin{array}{c}
w_{1} \\
w_{2} \\
\vdots \\
w_{5}
\end{array}\right] \circ\left[\begin{array}{c}
D_{1} \\
D_{2} \\
\vdots \\
D_{5}
\end{array}\right]\right\},
$$

where $\left[w_{\mathrm{i}}\right]_{t}$ is the weight vector of the second-level risk evaluation index at $t$, and $\left[D_{\mathrm{i}}\right]_{t}$ is the fuzzy evaluation matrix of the second-level risk evaluation index at $t .[w]_{t}$ is the weight vector of the first-level risk evaluation index at $t,\left[B_{\mathrm{i}}\right]_{t}$ is the fuzzy evaluation matrix of the first-level risk evaluation index at $t$, and $[B]_{t}$ is the second-level comprehensive evaluation matrix at $t .\left[B_{\mathrm{i}}\right]_{t}$ and $[B]_{t}$ reflect the result of the fuzzy comprehensive assessment. $\bigcirc$ is the fuzzy composition operator, and the operations of the fuzzy composition operator can be divided into three categories: taking the larger; taking the smaller; and weighted average.

$[B]_{t}$ is the final calculation result of the fuzzy comprehensive assessment method, and its essence is still a fuzzy vector. The result should be clarified in order to analyze the risk evaluation and level. The commonly used methods are the maximum membership, median, and subsection assignment methods.

The calculated results of the fuzzy comprehensive assessment can be obtained by the fuzzy synthesis of the dynamic weights of the risk evaluation indexes and the fuzzy evaluation matrix. Among them, the fuzzy synthesis operator adopts the weighted average operator, which can comprehensively consider the effects of various factors. After the calculation, the subsection assignment method, which can comprehensively consider the calculation results of each risk level, is used to verify the calculation results. 
Table 7. Fuzzy evaluation matrix of risk evaluation index in each construction stage.

\begin{tabular}{|c|c|c|c|c|c|c|c|c|c|c|c|c|c|c|c|c|c|c|c|c|c|c|c|c|c|}
\hline \multirow{3}{*}{$\begin{array}{l}\text { Second- } \\
\text { Level } \\
\text { Indexes }\end{array}$} & \multicolumn{25}{|c|}{ Construction Stage } \\
\hline & \multicolumn{5}{|c|}{ Initial Stage } & \multicolumn{5}{|c|}{ Stage 1} & \multicolumn{5}{|c|}{ Stage 2} & \multicolumn{5}{|c|}{ Stage 3} & \multicolumn{5}{|c|}{ Stage 4} \\
\hline & 1 & 2 & 3 & 4 & 5 & 1 & 2 & 3 & 4 & 5 & 1 & 2 & 3 & 4 & 5 & 1 & 2 & 3 & 4 & 5 & 1 & 2 & 3 & 4 & 5 \\
\hline $\mathrm{U}_{12}$ & 0 & 0 & 0 & 1 & 0 & 0 & 0 & 0 & 1 & 0 & 0 & 0 & 0 & 0.5 & 0.5 & 0 & 0 & 0 & 1 & 0 & 0 & 0 & 0 & 0.5 & 0.5 \\
\hline $\mathrm{U}_{13}$ & 0 & 0 & 0 & 1 & 0 & 0 & 0 & 0 & 1 & 0 & 0 & 0 & 0 & 0 & 1 & 0 & 0 & 0 & 0.6 & 0.4 & 0 & 0 & 0 & 0 & 1 \\
\hline $\mathrm{U}_{14}$ & 0 & 0 & 1 & 0 & 0 & 0 & 0 & 0.3 & 0.7 & 0 & 0 & 0 & 0.7 & 0.3 & 0 & 0 & 0 & 1 & 0 & 0 & 0 & 0 & 0.5 & 0.5 & 0 \\
\hline $\mathrm{U}_{15}$ & 0 & 0 & 0 & 1 & 0 & 0 & 0 & 1 & 0 & 0 & 0 & 0 & 0 & 0.5 & 0.5 & 0 & 0 & 0.8 & 0.2 & 0 & 0 & 0 & 0 & 0.7 & 0.3 \\
\hline $\mathrm{U}_{22}$ & 0 & 0 & 0 & 1 & 0 & 0 & 0 & 0.3 & 0.7 & 0 & 0 & 0 & 0 & 0.5 & 0.5 & 0 & 0 & 0 & 1 & 0 & 0 & 0 & 0 & 0.5 & 0.5 \\
\hline $\mathrm{U}_{23}$ & 0 & 0 & 0 & 0.5 & 0.5 & 0 & 0 & 1 & 0 & 0 & 0 & 0 & 0 & 0.3 & 0.7 & 0 & 0 & 0 & 0.3 & 0.7 & 0 & 0.8 & 0.2 & 0 & 0 \\
\hline $\mathrm{U}_{24}$ & 0 & 0 & 0 & 1 & 0 & 0 & 0 & 0.3 & 0.7 & 0 & 0 & 0 & 0 & 1 & 0 & 0 & 0 & 0.3 & 0.7 & 0 & 0 & 0.8 & 0.2 & 0 & 0 \\
\hline $\mathrm{U}_{31}$ & 0 & 0 & 0 & 0.5 & 0.5 & 0 & 0 & 0.3 & 0.7 & 0 & 0 & 0 & 0 & 0.3 & 0.7 & 0 & 0 & 0 & 1 & 0 & 0 & 0 & 0 & 0.9 & 0.1 \\
\hline $\mathrm{U}_{32}$ & 0 & 0 & 0 & 1 & 0 & 0 & 0 & 1 & 0 & 0 & 0 & 0 & 0 & 0.4 & 0.6 & 0 & 0 & 0 & 0.5 & 0.5 & 0 & 0 & 0.7 & 0.3 & 0 \\
\hline $\mathrm{U}_{33}$ & 0 & 1 & 0 & 0 & 0 & 0 & 1 & 0 & 0 & 0 & 0 & 1 & 0 & 0 & 0 & 0 & 1 & 0 & 0 & 0 & 0 & 0 & 1 & 0 & 0 \\
\hline $\mathrm{U}_{34}$ & 0 & 1 & 0 & 0 & 0 & 0 & 1 & 0 & 0 & 0 & 0 & 1 & 0 & 0 & 0 & 0 & 1 & 0 & 0 & 0 & 0 & 0 & 0.8 & 0.2 & 0 \\
\hline $\mathrm{U}_{35}$ & 0 & 0 & 0 & 0.8 & 0.2 & 0 & 0 & 0 & 1 & 0 & 0 & 0 & 0 & 0.2 & 0.8 & 0 & 0 & 0 & 1 & 0 & 0 & 0.5 & 0.3 & 0 & 0 \\
\hline $\mathrm{U}_{36}$ & 0 & 0 & 1 & 0 & 0 & 0 & 1 & 0 & 0 & 0 & 0 & 0 & 1 & 0 & 0 & 0 & 0 & 1 & 0 & 0 & 0 & 0 & 0.8 & 0.2 & 0 \\
\hline $\mathrm{U}_{42}$ & 0 & 0 & 1 & 0 & 0 & 0.1 & 0.9 & 0 & 0 & 0 & 0 & 0.3 & 0.7 & 0 & 0 & 0 & 0.2 & 0.8 & 0 & 0 & 0 & 0.3 & 0.7 & 0 & 0 \\
\hline $\mathrm{U}_{43}$ & 0 & 1 & 0 & 0 & 0 & 0 & 0 & 0.5 & 0.5 & 0 & 0 & 1 & 0 & 0 & 0 & 0 & 1 & 0 & 0 & 0 & 0 & 0.5 & 0.5 & 0 & 0 \\
\hline $\mathrm{U}_{44}$ & 0 & 0 & 1 & 0 & 0 & 0 & 0 & 1 & 0 & 0 & 0 & 0 & 0.9 & 0.1 & 0 & 0 & 0 & 0.3 & 0.7 & 0 & 0 & 0 & 1 & 0 & 0 \\
\hline $\mathrm{U}_{45}$ & 0 & 1 & 0 & 0 & 0 & 0 & 0.5 & 0.5 & 0 & 0 & 0 & 1 & 0 & 0 & 0 & 0 & 1 & 0 & 0 & 0 & 0 & 0.9 & 0.1 & 0 & 0 \\
\hline $\mathrm{U}_{51}$ & 0 & 0.4 & 0.6 & 0 & 0 & 0 & 0 & 1 & 0 & 0 & 0 & 0.3 & 0.7 & 0 & 0 & 0 & 0.9 & 0.1 & 0 & 0 & 0 & 0.5 & 0.5 & 0 & 0 \\
\hline $\mathrm{U}_{52}$ & 0.1 & 0.9 & 0 & 0 & 0 & 0 & 0.5 & 0.5 & 0 & 0 & 0 & 1 & 0 & 0 & 0 & 0 & 0.9 & 0.1 & 0 & 0 & 0 & 0 & 0 & 0 & 1 \\
\hline $\mathrm{U}_{53}$ & 0 & 0 & 1 & 0 & 0 & 0 & 0 & 1 & 0 & 0 & 0 & 1 & 0 & 0 & 0 & 0 & 0.2 & 0.8 & 0 & 0 & 0 & 0 & 0 & 0 & 1 \\
\hline $\mathrm{U}_{54}$ & 0 & 1 & 0 & 0 & 0 & 0 & 0.8 & 0.2 & 0 & 0 & 0 & 1 & 0 & 0 & 0 & 0 & 1 & 0 & 0 & 0 & 0 & 0 & 0 & 0 & 1 \\
\hline $\mathrm{U}_{55}$ & 0 & 1 & 0 & 0 & 0 & 0 & 1 & 0 & 0 & 0 & 0 & 1 & 0 & 0 & 0 & 0.3 & 0.7 & 0 & 0 & 0 & 0 & 0 & 0 & 0.3 & 0.7 \\
\hline $\mathrm{U}_{61}$ & & & & & & 0 & 1 & 0 & 0 & 0 & 0 & 0 & 0 & 1 & 0 & 0 & 0 & 1 & 0 & 0 & 0 & 0 & 0 & 1 & 0 \\
\hline $\mathrm{U}_{62}$ & & & & & & 1 & 0 & 0 & 0 & 0 & 0 & 0 & 0 & 1 & 0 & 0 & 0 & 1 & 0 & 0 & 0 & 0 & 1 & 0 & 0 \\
\hline $\mathrm{U}_{63}$ & & & & & & 0 & 1 & 0 & 0 & 0 & 0 & 0 & 0 & 1 & 0 & 0 & 0 & 1 & 0 & 0 & 0 & 0 & 1 & 0 & 0 \\
\hline $\mathrm{U}_{64}$ & & & & & & 1 & 0 & 0 & 0 & 0 & 0 & 0 & 0.2 & 0.8 & 0 & 0 & 0 & 0 & 1 & 0 & 0 & 0 & 0 & 1 & 0 \\
\hline $\mathrm{U}_{65}$ & & & & & & 1 & 0 & 0 & 0 & 0 & 0 & 0 & 1 & 0 & 0 & 0 & 0 & 0.5 & 0.5 & 0 & 0 & 0 & 0.5 & 0.5 & 0 \\
\hline
\end{tabular}




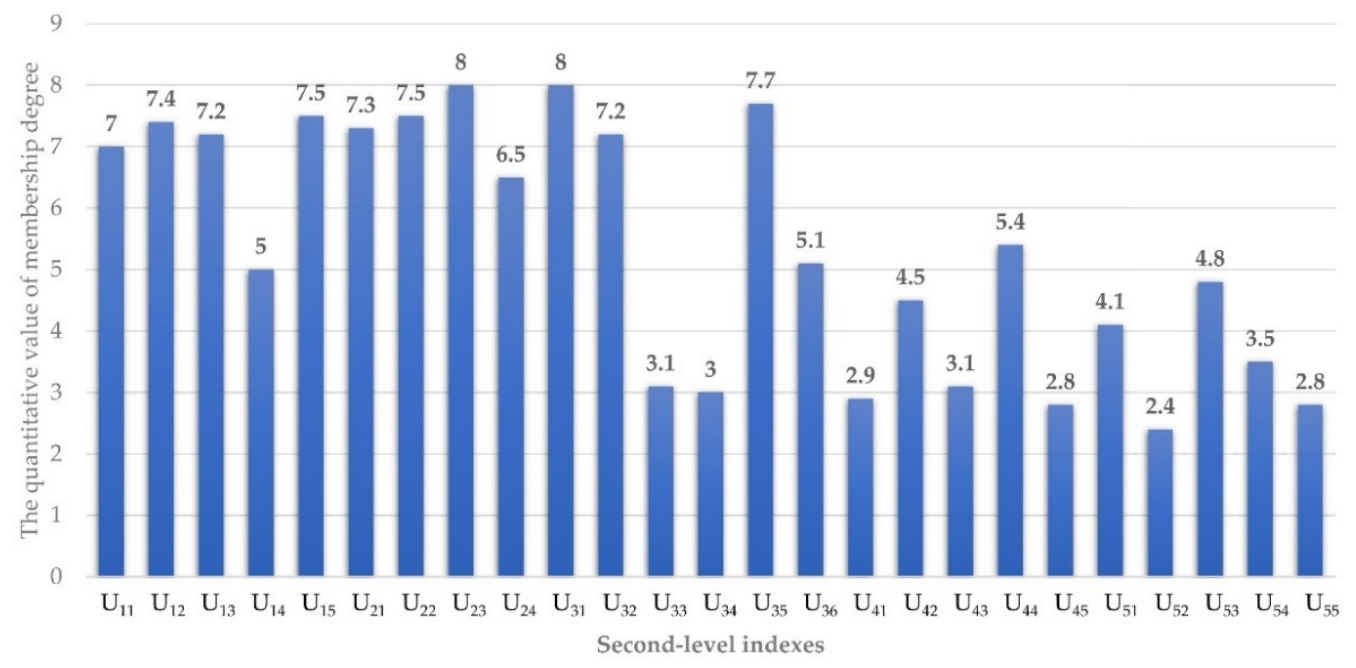

(a)

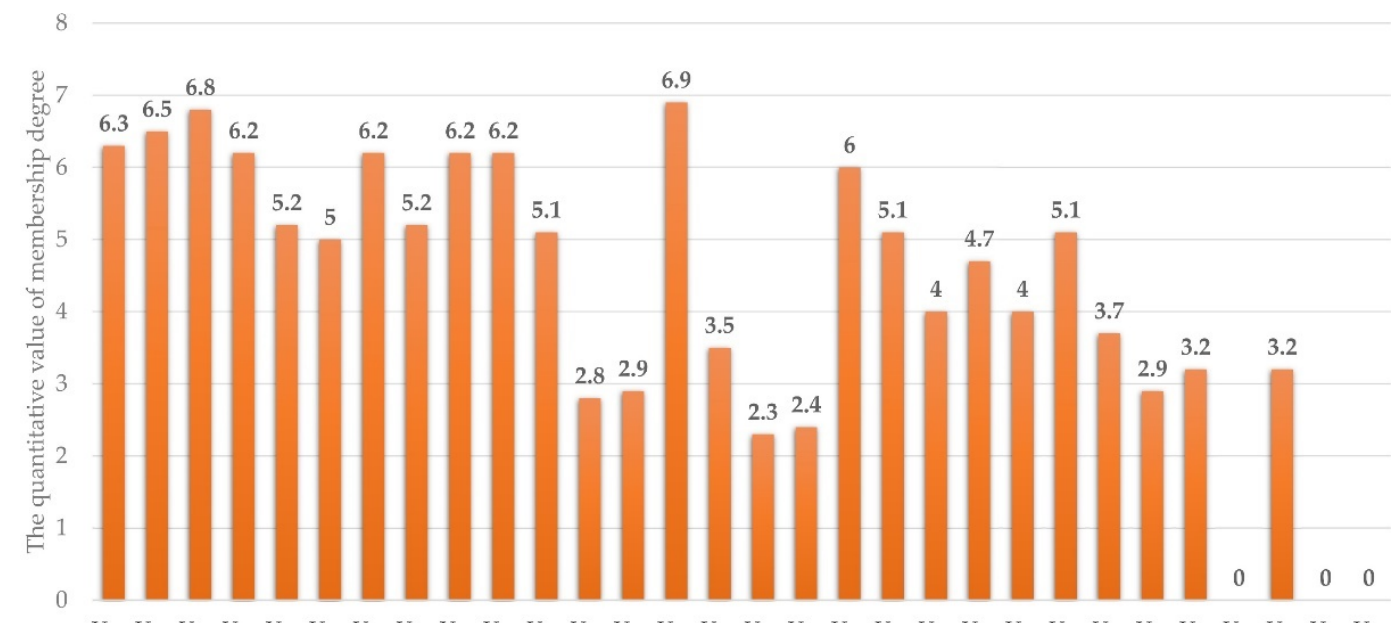

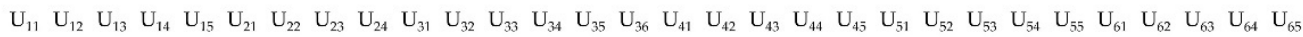
Second-level indexes

(b)

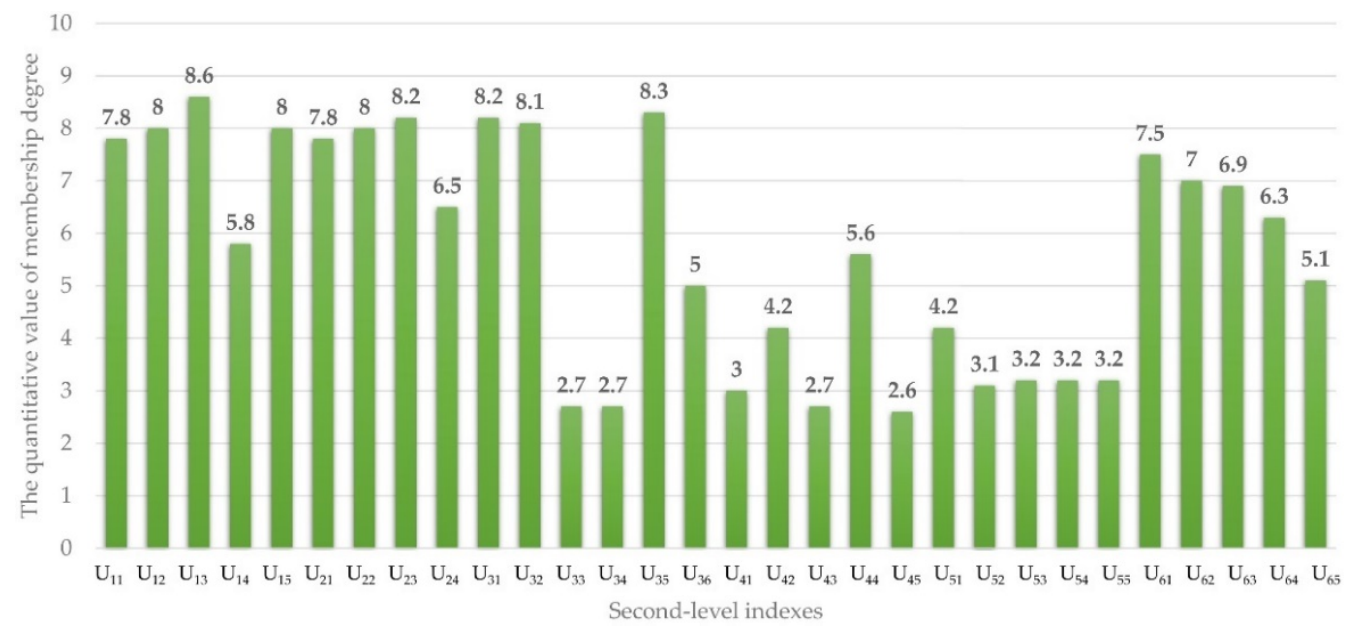

(c)

Figure 4. Cont. 


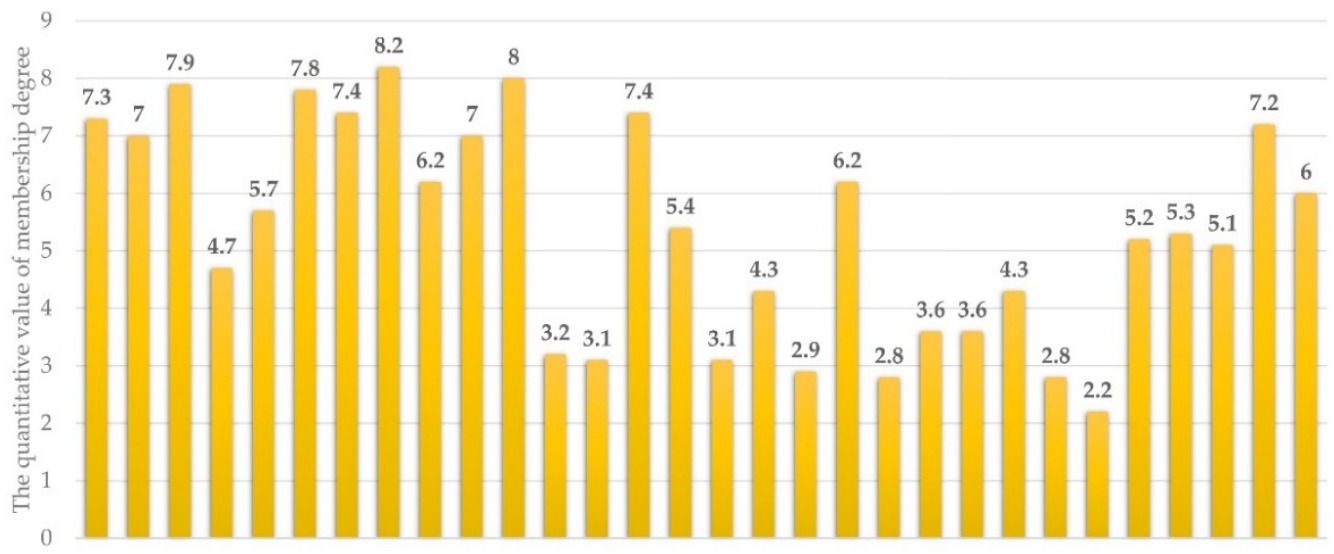

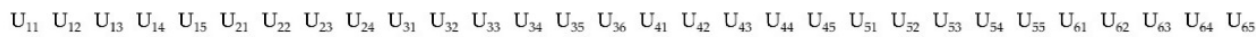
Second-level indexes

(d)

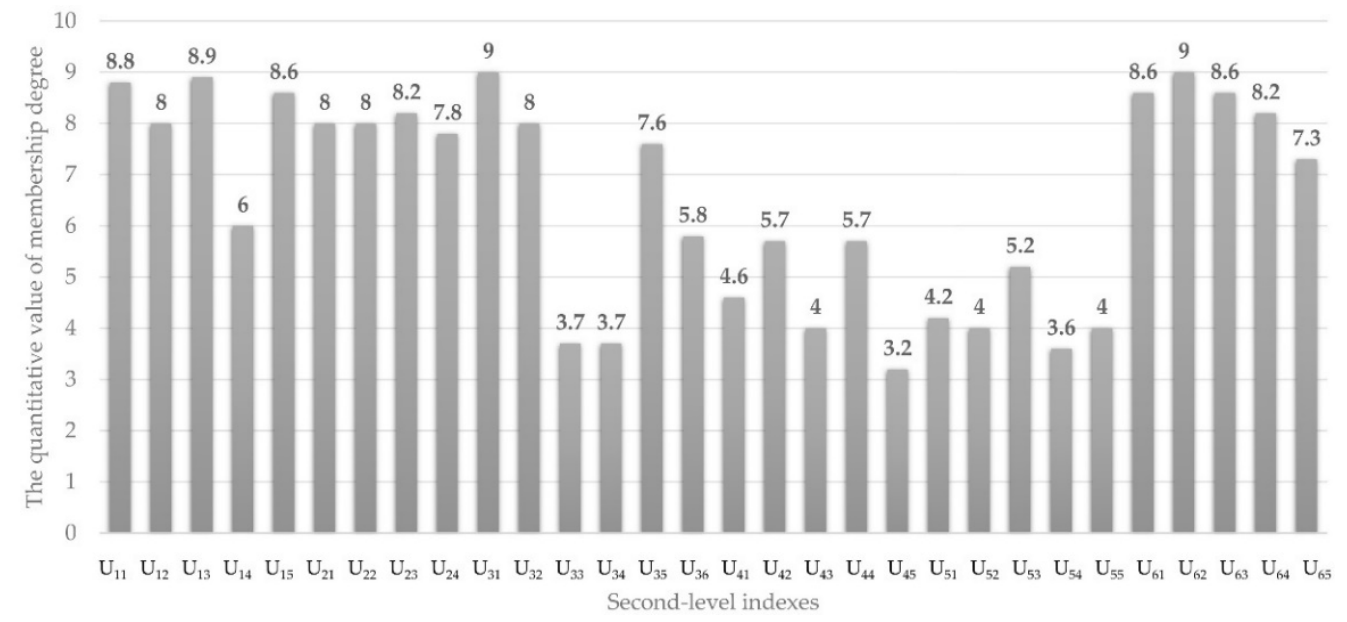

(e)

Figure 4. The quantitative value of risk evaluation indexes membership degree at each stage: (a) Initial stage; (b) Stage 1; (c) Stage 2; (d) Stage 3; (e) Stage 4.

\subsubsection{First-Level Fuzzy Comprehensive Assessment}

The weighted average operator was used to synthesize the obtained dynamic weight of the second-level risk evaluation index and the fuzzy evaluation matrix to obtain the first-level evaluation matrix at each stage, as shown in Table 8.

\subsubsection{Second-Level Fuzzy Comprehensive Assessment}

According to the weight of the first-level risk evaluation index and the first-level evaluation matrix of each stage, the evaluation matrix composed of the second-level comprehensive evaluation vectors of each stage can be obtained by fuzzy calculation, as shown in Table 9. 
Table 8. First-level evaluation matrix.

\begin{tabular}{|c|c|c|c|c|c|c|}
\hline \multirow{2}{*}{ Stage } & \multirow{2}{*}{ Risk Evaluation Indexes } & \multicolumn{5}{|c|}{ Assessment Results } \\
\hline & & 1 & 2 & 3 & 4 & 5 \\
\hline \multirow{5}{*}{ Initial stage } & Engineering geology and hydrogeology $\mathrm{U}_{1}$ & 0.00 & 0.00 & 0.10 & 0.90 & 0.00 \\
\hline & Surrounding construction environment $\mathrm{U}_{2}$ & 0.00 & 0.00 & 0.00 & 0.93 & 0.07 \\
\hline & Overall engineering design $\mathrm{U}_{3}$ & 0.00 & 0.46 & 0.08 & 0.32 & 0.14 \\
\hline & Engineering construction technology $\mathrm{U}_{4}$ & 0.00 & 0.78 & 0.22 & 0.00 & 0.00 \\
\hline & Engineering construction management $\mathrm{U}_{5}$ & 0.04 & 0.67 & 0.29 & 0.00 & 0.00 \\
\hline \multirow{6}{*}{ Stage 1} & Engineering geology and hydrogeology $\mathrm{U}_{1}$ & 0.00 & 0.00 & 0.31 & 0.69 & 0.00 \\
\hline & Surrounding construction environment $\mathrm{U}_{2}$ & 0.00 & 0.00 & 0.71 & 0.29 & 0.00 \\
\hline & Overall engineering design $\mathrm{U}_{3}$ & 0.00 & 0.50 & 0.23 & 0.27 & 0.00 \\
\hline & Engineering construction technology $\mathrm{U}_{4}$ & 0.05 & 0.32 & 0.40 & 0.23 & 0.00 \\
\hline & Engineering construction management $\mathrm{U}_{5}$ & 0.00 & 0.39 & 0.61 & 0.00 & 0.00 \\
\hline & Monitoring measurement data $\mathrm{U}_{6}$ & 0.00 & 1.00 & 0.00 & 0.00 & 0.00 \\
\hline \multirow{6}{*}{ Stage 2} & Engineering geology and hydrogeology $\mathrm{U}_{1}$ & 0.00 & 0.00 & 0.06 & 0.48 & 0.46 \\
\hline & Surrounding construction environment $\mathrm{U}_{2}$ & 0.00 & 0.00 & 0.00 & 0.59 & 0.41 \\
\hline & Overall engineering design $\mathrm{U}_{3}$ & 0.00 & 0.37 & 0.12 & 0.16 & 0.35 \\
\hline & Engineering construction technology $\mathrm{U}_{4}$ & 0.00 & 0.78 & 0.21 & 0.01 & 0.00 \\
\hline & Engineering construction management $\mathrm{U}_{5}$ & 0.00 & 0.83 & 0.17 & 0.00 & 0.00 \\
\hline & Monitoring measurement data $\mathrm{U}_{6}$ & 0.00 & 0.00 & 0.11 & 0.89 & 0.00 \\
\hline \multirow{6}{*}{ Stage 3} & Engineering geology and hydrogeology $\mathrm{U}_{1}$ & 0.00 & 0.00 & 0.26 & 0.69 & 0.05 \\
\hline & Surrounding construction environment $\mathrm{U}_{2}$ & 0.00 & 0.00 & 0.04 & 0.70 & 0.26 \\
\hline & Overall engineering design $\mathrm{U}_{3}$ & 0.00 & 0.34 & 0.10 & 0.44 & 0.12 \\
\hline & Engineering construction technology $\mathrm{U}_{4}$ & 0.00 & 0.75 & 0.16 & 0.09 & 0.00 \\
\hline & Engineering construction management $\mathrm{U}_{5}$ & 0.03 & 0.79 & 0.18 & 0.00 & 0.00 \\
\hline & Monitoring measurement data $\mathrm{U}_{6}$ & 0.00 & 0.00 & 0.80 & 0.20 & 0.00 \\
\hline \multirow{6}{*}{ Stage 4} & Engineering geology and hydrogeology $\mathrm{U}_{1}$ & 0.00 & 0.00 & 0.04 & 0.12 & 0.84 \\
\hline & Surrounding construction environment $\mathrm{U}_{2}$ & 0.00 & 0.00 & 0.00 & 0.50 & 0.50 \\
\hline & Overall engineering design $\mathrm{U}_{3}$ & 0.00 & 0.15 & 0.10 & 0.23 & 0.52 \\
\hline & Engineering construction technology $\mathrm{U}_{4}$ & 0.00 & 0.32 & 0.55 & 0.05 & 0.00 \\
\hline & Engineering construction management $\mathrm{U}_{5}$ & 0.00 & 0.42 & 0.58 & 0.00 & 0.00 \\
\hline & Monitoring measurement data $\mathrm{U}_{6}$ & 0.00 & 0.00 & 0.00 & 0.15 & 0.86 \\
\hline
\end{tabular}

Table 9. Risk assessment results of each stage.

\begin{tabular}{cccccc}
\hline \multirow{2}{*}{ Stage } & \multicolumn{5}{c}{ Assessment Results } \\
\cline { 2 - 6 } & $\mathbf{1}$ & $\mathbf{2}$ & $\mathbf{3}$ & $\mathbf{4}$ & $\mathbf{5}$ \\
\hline Initial stage & 0.01 & 0.32 & 0.12 & 0.51 & 0.05 \\
Stage 1 & 0.01 & 0.41 & 0.29 & 0.29 & 0.00 \\
Stage 2 & 0.00 & 0.23 & 0.11 & 0.44 & 0.23 \\
Stage 3 & 0.00 & 0.22 & 0.31 & 0.40 & 0.07 \\
Stage 4 & 0.00 & 0.10 & 0.14 & 0.18 & 0.58 \\
\hline
\end{tabular}

The calculation results were analyzed using the subsection assignment method. The risk evaluation level was divided into five grades: safe; relatively safe; generally safe; relatively dangerous; and dangerous. As such, a certain quantitative score was given to each level, as shown in Figure 5. The risk level was divided into five levels according to the range where the score was calculated, as shown in Figure 6. 


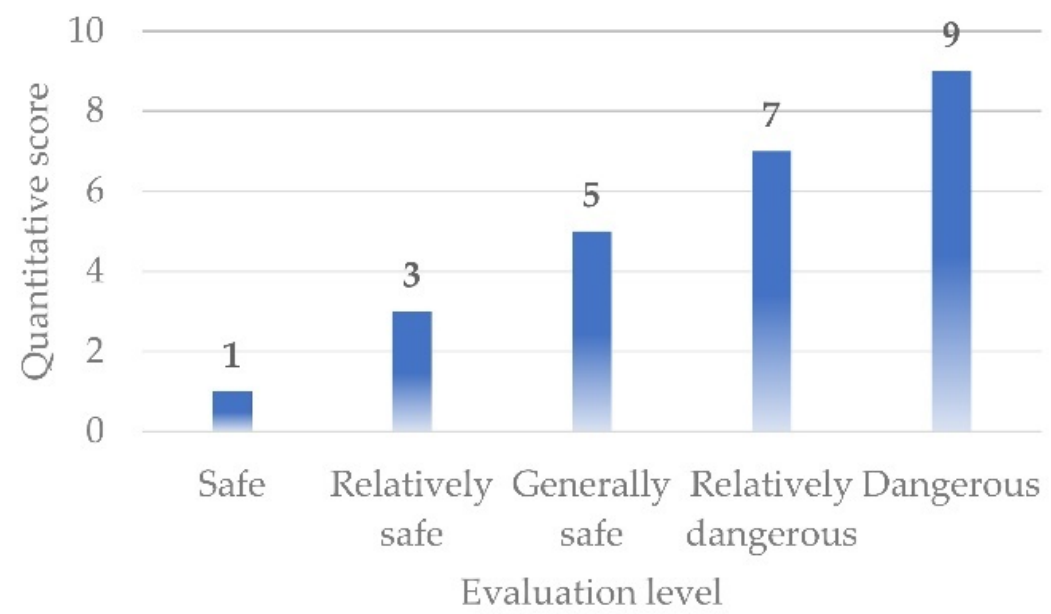

Figure 5. Relationship between evaluation level and quantitative score.

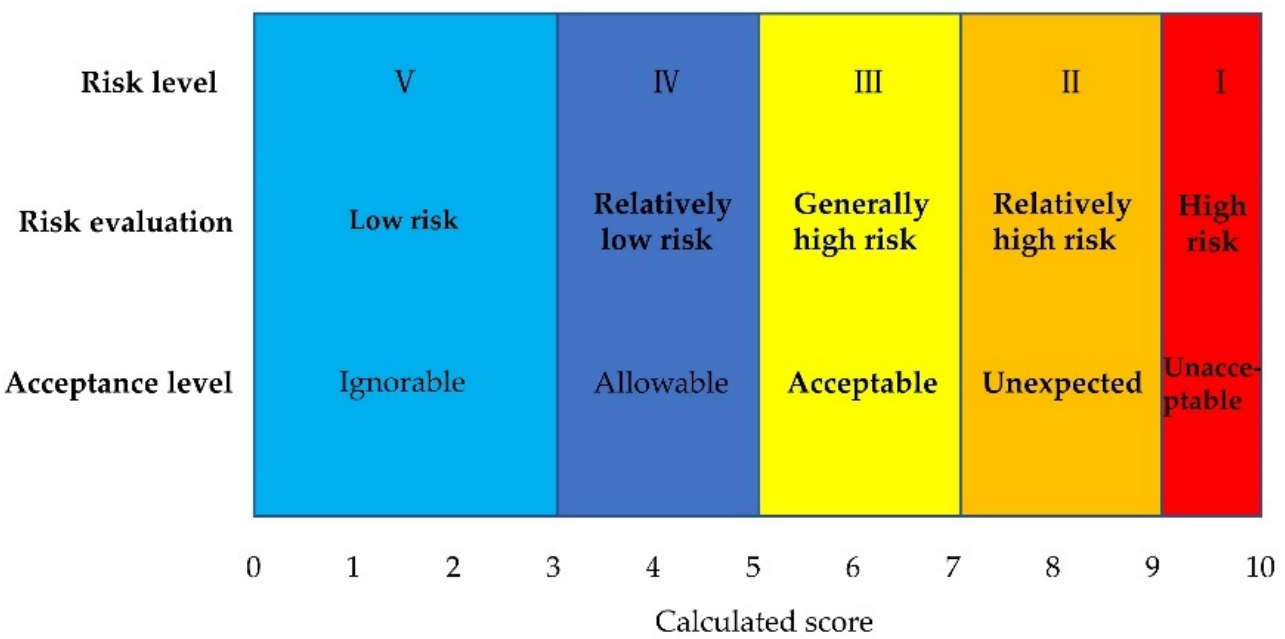

Figure 6. Risk level and calculated score.

As shown in Figure 6, when $Q \leq 3$, the risk level was class $\mathrm{V}$, indicating a very low level of risk and a negligible acceptance degree at this stage, where risk treatment measures were unnecessary. When $3 \leq Q<5$, the risk level was IV, indicating low risk and a tolerable acceptance level at this stage. The risks should be monitored at all times for all the monitoring indicators specified, however risk treatment measures were still unnecessary. When $5 \leq Q<7$, the risk level was III, indicating that the risk evaluation conclusion at this stage was a medium/acceptable risk, and the risk occurrence probability and risk loss were high, yet within the controllable range. This level of risk must be given attention, and necessary risk treatment measures should be taken while strengthening the monitoring. When $7 \leq Q<9$, the risk level was II. This kind of risk is major and is not expected to occur. The probability of risk occurrence was high, and the risk loss was large, requiring great attention. When $Q \geq 9$, the risk level was I, which is unacceptable. The risk occurrence probability was greater than that of the level II risk, and the loss was unacceptable. A series of measures must be taken in advance to avoid its occurrence or further deterioration.

If the assessment result is at level I risk, the construction shall be stopped immediately, and comprehensive rectification shall be carried out to reduce the risk assessment level. Through the assignment calculation, the pre-construction preparation stage of the shallowburied large-span double-arch tunnel and the risk evaluation score $(Q)$ of each stage during the construction process were finally obtained. Then, the risk evaluation and risk acceptance levels of each stage can be obtained by referring to Figure 6 . The specific results are shown in Figure 7. 


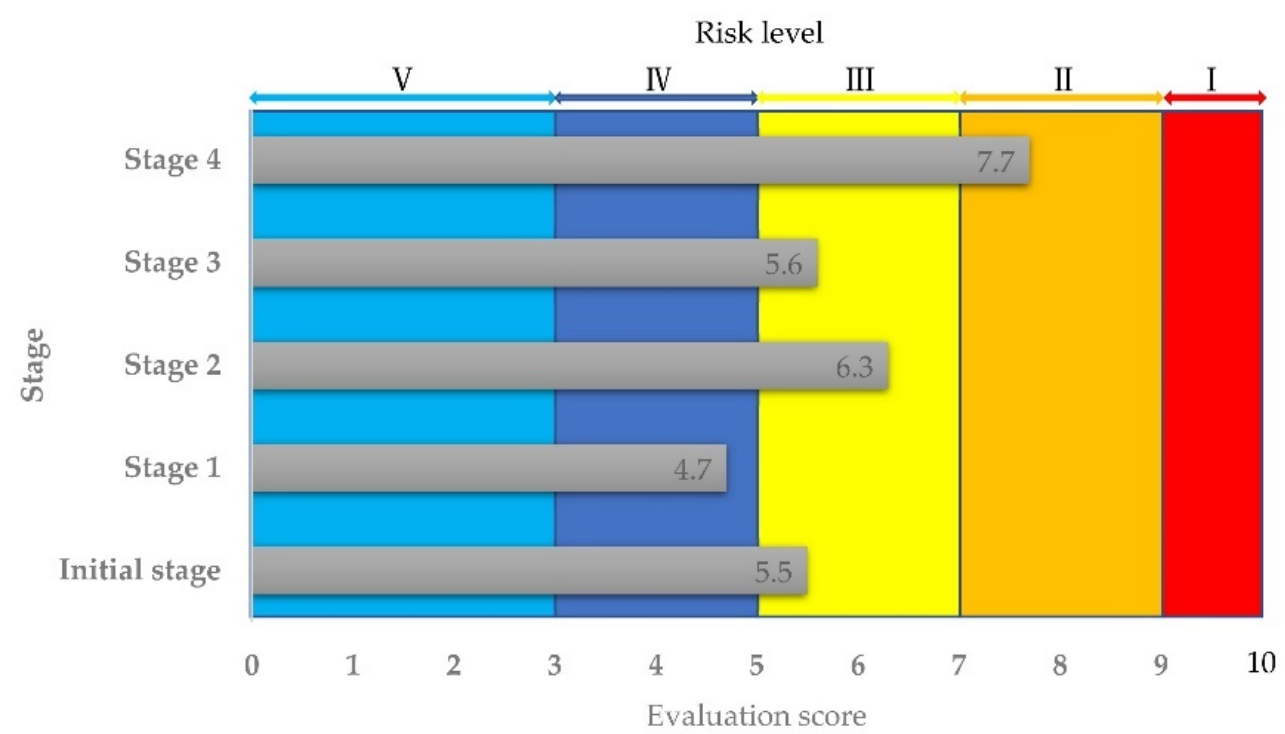

Figure 7. Dynamic risk evaluation results of each stage.

The risk evaluation result in the initial stage was level III, indicating that the tunnel construction project was generally at a medium risk level, and the formulation of perfect risk prevention policies and risk treatment measures was therefore necessary, and the monitoring items required by the specification (such as ground settlement, pipeline deformation, vault settlement, surrounding rock pressure, and anchor rod axial force) required monitoring. The risk evaluation result of stage 1 (ground and surrounding rock reinforcement) was level IV. The risk at this stage was low, requiring no risk treatment measures. The risk evaluation results of stage 2 (pilot tunnel excavation) and stage 3 (main tunnel single tunnel excavation) were both level III. During the construction process, attention must be given to the changes in monitoring data to avoid risks in a timely manner. The risk evaluation result of stage 4 (main tunnel double tunnel excavation) was level II, and the risk level was relatively high. The risk at this stage must be given great attention. The monitoring encrypted the monitoring control points, improved the monitoring frequency, and reduced the risk by strengthening management and risk treatment measures.

The risk assessment result of the traditional static fuzzy comprehensive assessment method for this project was level III. The risk prevention policies and risk treatment measures formulated according to level III risk during construction undoubtedly caused the waste of social resources in the ground and surrounding rock reinforcement stage and led to insufficient risk prevention awareness and protection measures in the main tunnel double tunnel excavation stage. The dynamic construction safety risk assessment based on the fuzzy comprehensive assessment method is more timely, accurate, and reasonable than the traditional assessment method. Dynamic construction safety risk assessment can accurately grasp the potential risks in each construction stage, reduce the occurrence of safety accidents in the construction process, and save social resources.

\section{Risk Control of Shallow Buried Large-Span Double-Arch Tunnel}

According to the construction risk assessment results, the construction risk was high in the main tunnel excavation and pilot tunnel excavation stages. Therefore, reasonable, effective, and feasible measures should be taken to reduce the probability of risk occurrence and the loss caused by risk. The corresponding risk control measures include engineering geology and hydrogeology, surrounding environment, engineering design scheme, and engineering construction and management.

For the construction risks of shallow-buried large-span double-arch tunnels caused by engineering geology and hydrogeological conditions, the following risk control measures can be taken: (i) Strengthening of the advanced geological prediction. During construction, the geological conditions in front of the tunnel excavation face shall be explored by flat 
drilling, geophysical exploration, and other methods, and corresponding preventive and treatment measures shall be taken according to the detection results, as shown in Figure 8; (ii) Strengthening of the advance support measures. The advance long pipe shed shall be erected at the portal, as shown in Figure 9. During tunnel excavation, the advanced long pipe shed and small conduit shall be simultaneously constructed to ensure the stability of the surrounding rock near the tunnel excavation surface and prevent collapse accidents; (iii) Strengthening the ground reinforcement. To control the nearby ground settlement deformation caused by tunnel construction and the structural deformation caused by the vehicle load on the underground tunnel, the subgrade slope in the Xinghu section above the tunnel excavation area is reinforced by grouting; (iv) Strengthening the monitoring and the measurement. During tunnel excavation, the monitoring and measurement of the ground, vault, groundwater level, surrounding pipelines, soil pressure, and support structure shall be strengthened, and the measured data shall be fed back to the construction and design in a timely manner to adjust the construction method, the construction process, the support structure type, and the design parameters; (v) Full-section curtain grouting is considered as reinforcement for the fault fracture zone. A fault fracture zone exists at BK17 + 890 BK17 + 930 in the underground excavation section of the project. The rock mass strength is lower than the surrounding rock mass, and the stability is poor. When the tunnel is excavated to this section, groundwater leakage and tunnel collapse may occur. Full-section curtain grouting reinforcement technology is used to send the prepared cement grout to the rock mass in front of the excavation through the grouting borehole laid in advance. After the slurry is diffused and solidified, the physical and mechanical properties of the surrounding rock can be improved, as shown in Figure 10.

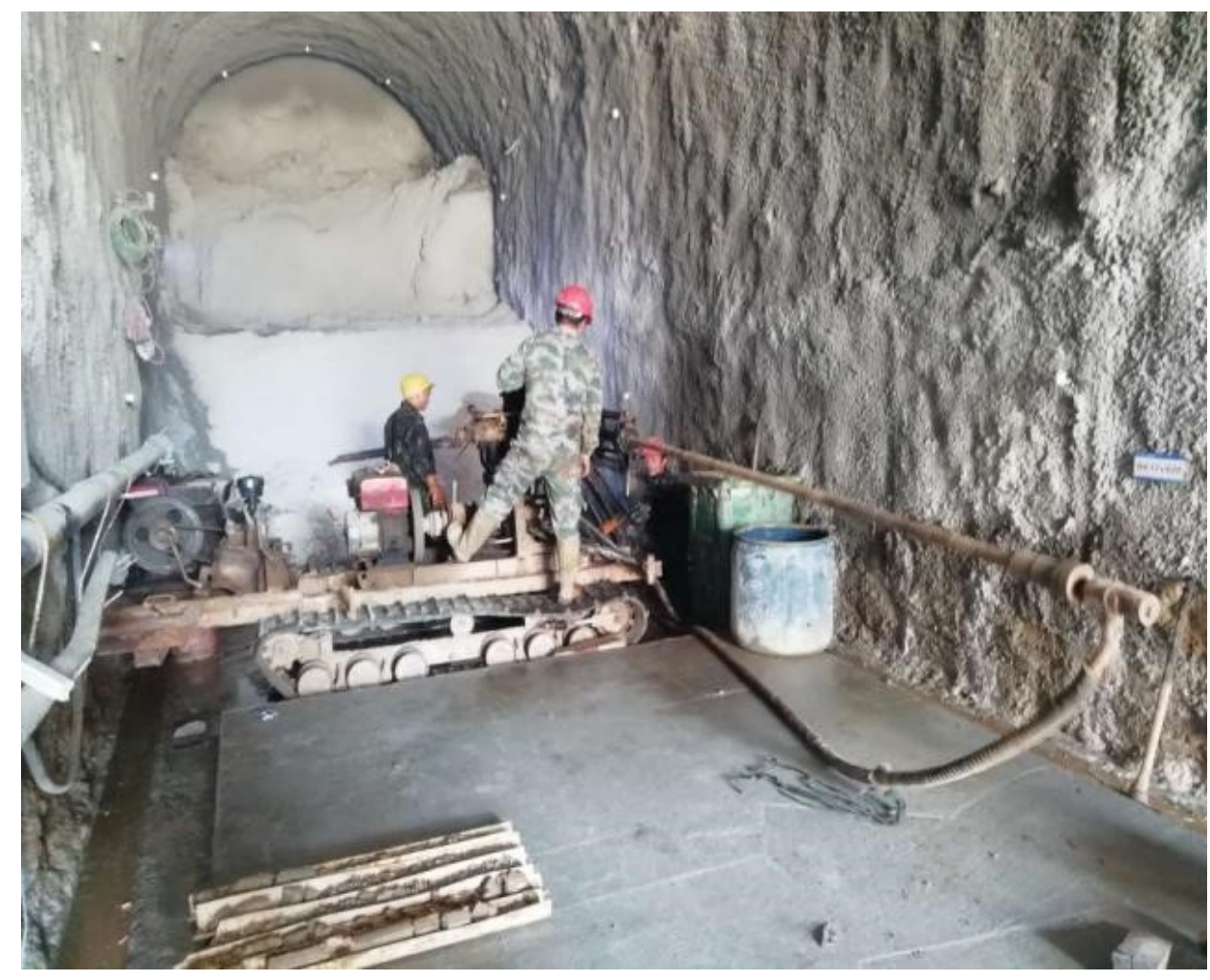

Figure 8. Advance geological prediction by drilling method. 


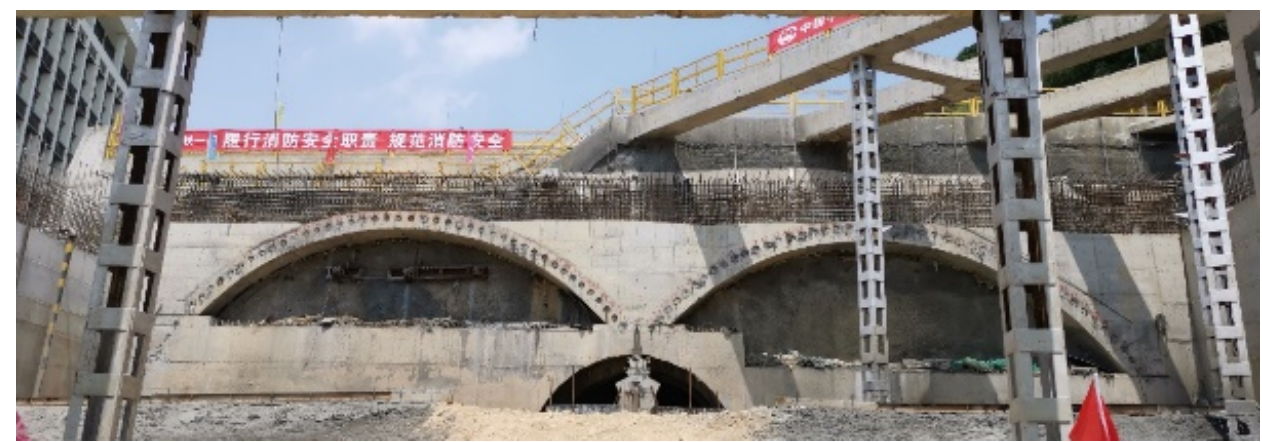

Figure 9. Advance long pipe shed.

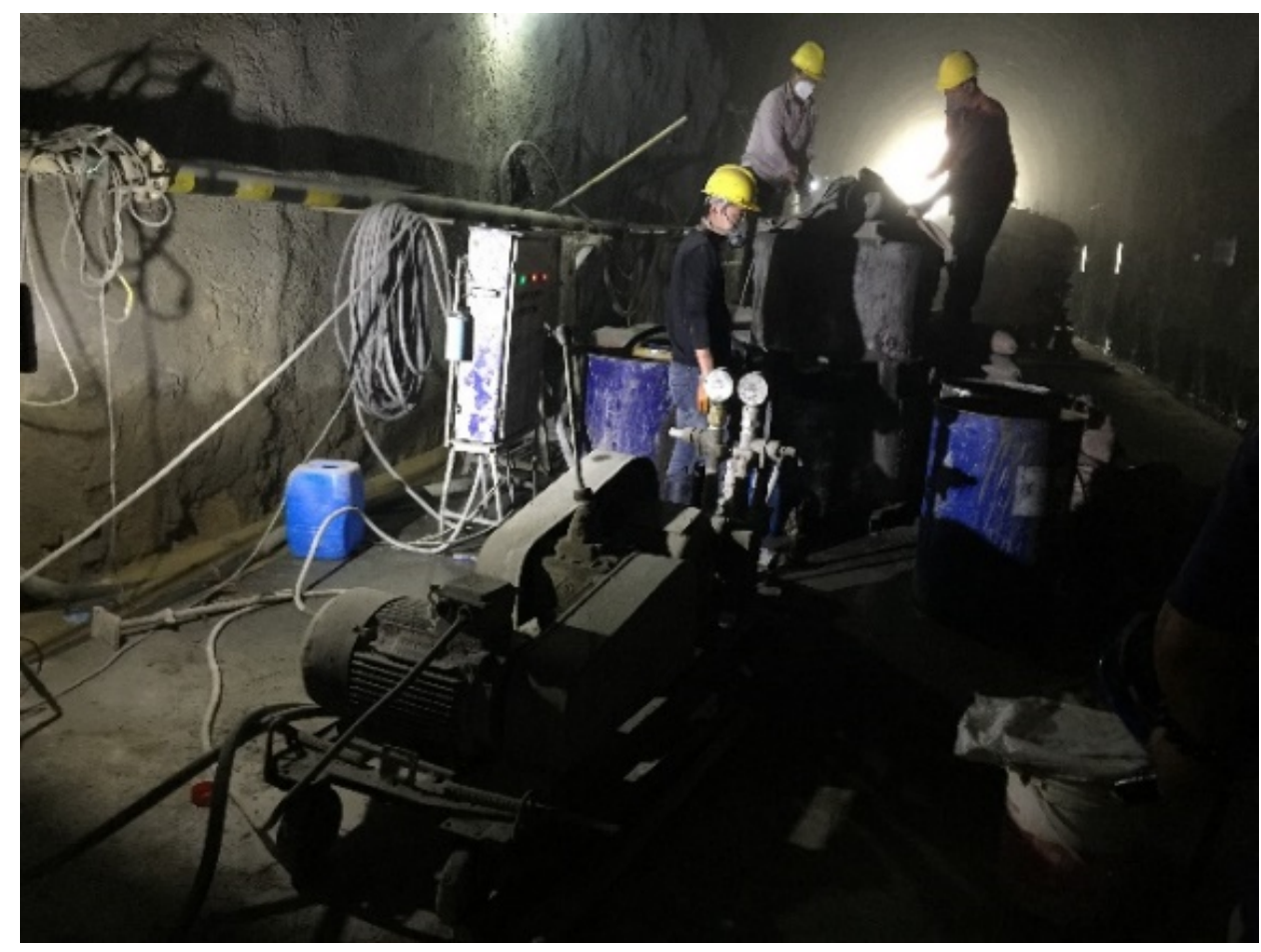

Figure 10. Full section curtain grouting.

To ensure that the construction of shallow tunnels can be carried out safely and smoothly, under the premise of the continued normal use of the surrounding buildings, expressways, and underground pipelines, the following measures can be taken for risk control: (i) Detailed underground pipeline protection measures and an emergency treatment plan should be formulated before tunnel construction, and sufficient anti-collapse and anti-leakage materials should be prepared to ensure that small leakage or collapse events can be handled in a timely manner to prevent worse accidents; (ii) Before construction, preventive measures must be taken with regard to the surrounding buildings and monitoring schemes during the construction period shall be formulated, and the settlement and deformation of surrounding buildings shall be investigated and monitored in a timely manner during construction; (iii) The subgrade slope surface and slope toe within the influence range of tunnel construction shall be reinforced by surface vertical grouting in advance, and the measures of transverse grouting reinforcement shall be adopted within the main traffic lane.

The reliability of the engineering design scheme determines whether the project can be carried out smoothly. In terms of the engineering design scheme, the following risk control measures can be taken: (i) Tunnel location selection, linear design, cross-section design, and support structure design should be carried out in strict accordance with the 
highway tunnel design specifications; (ii) Independent design schemes should be specially written for the important and difficult parts of the project, such as the ground surface reinforcement design plan, three-pilot-tunnel excavation and support design plan, and partition wall force optimization plan; (iii) Experts in highway tunnels must be organized to review the design scheme and drawings. In the field implementation process, dynamic analysis of data must be carried out in combination with results monitoring, and the design scheme should be modified and improved in real time according to the data analysis results to realize dynamic design.

In terms of project construction and management, relevant measures can be taken to strengthen the quality management, the organization management, and the safety management. The specific measures are as follows: (i) During construction, the excavation step distance shall be strictly controlled to approximately $2 \mathrm{~m}$ to prevent over excavation and under excavation. The distance between the upper steps of the three pilot tunnels should be kept at $20 \mathrm{~m}$, and the distance between the steps of the left and right main tunnels should be kept at $30 \mathrm{~m}$; (ii) After excavation, spray-anchor support should be carried out on time to reduce the stress release time of the surrounding rock and prevent the excessive deformation of the surrounding rock. After excavating the entire section, the invert and the secondary lining structure shall be constructed on time to make the supporting structure close into a ring as soon as possible; (iii) During tunnel excavation and supporting structure construction, the monitoring and measurement of the tunnel vault, clearance convergence, surrounding rock pressure, and supporting structure stress must be strengthened; (iv) The professional skills and quality construction training system of construction personnel must be improved.

During the construction period, all monitoring items ran normally, and no abnormal phenomenon or safety accidents occurred in the monitoring data. During the entire construction period of the shallow-buried tunnel, the driving conditions on Xinghu Road and the production and life of residents in the surrounding communities were unaffected. Finally, the project was successfully completed and accepted by the experts and relevant departments. The results show that the risk assessment methods used in this paper, the risk assessment results obtained, and the risk control measures proposed are reasonable and effective.

\section{Conclusions}

The main problem that faced this study was to overcome the deficiency of static risk assessment in an ultra-shallow-buried large-span double-arch tunnel. Xiamen Haicang double-arch tunnel was selected as an example, and the dynamic risk assessment was investigated for the ultra-shallow-buried large-span double-arch tunnel construction. Firstly, the construction process was divided into five stages, and the dynamic risk evaluation index system was established. Then the benchmark weight of the dynamic risk evaluation index was determined by AHP, and the weight of risk evaluation index was modified according to the construction stage. The risk evaluation indexes of the different construction stages were determined differently, and the weights of the risk evaluation indexes at the same level were changed during the construction process. The changes in weights affected the risk assessment results. Finally, the fuzzy comprehensive evaluation method was adopted to evaluate the risk of each stage, and the risk control measures were suggested. Therefore, the following conclusions can be drawn:

(1) Dividing the construction into different stages for risk evaluation according to the construction process and the key construction nodes improved the accuracy, timeliness, and rationality of the dynamic evaluation results;

(2) The risk assessment result of the main tunnel double tunnel excavation stage was level II, and the risk level was high. The risk assessment result of the ground and surrounding rock reinforcement stage was level IV, and the risk in this stage was low. The risk assessment results of the other stages were level III, and the risk was medium; 
(3) In the pre-construction preparation stage, comprehensive risk prevention policies and risk treatment measures should be formulated, and the monitoring of the items required by the specification during the project must be strengthened. No risk treatment measures were needed at the surface and surrounding rock reinforcement stage. During the construction of pilot tunnel and single tunnel excavation stages of the main tunnel, attention should be given to the changes in monitoring data. Great attention must be given to the risk in the excavation stage of the double tunnels of the main tunnel;

(4) The dynamic construction safety risk assessment based on the fuzzy comprehensive assessment method is more timely, accurate, and reasonable than the traditional assessment method. Dynamic risk assessment can promptly discover and prevent hidden dangers in the construction of shallow-buried tunnels.

Summarizing the research performed above, it can be concluded that the dynamic risk assessment of the Xiamen Haicang tunnel objectively reflected the dynamic risks. However, the dynamic risk identification of an ultra-shallow-buried and large-span double-arch tunnel construction maybe complex and uncertain. The influencing factors are normally determined by the experience of experts. The dynamic assessment currently depends on quantitative work. In the future, random field theory and probability method can be introduced to describe the risks, quantitative methods including artificial intelligence (AI), big data, digital technology, and AI monitoring can be involved in the dynamic assessment to identify the number of risk factors and improve the accuracy of risk assessment.

Author Contributions: J.W., Z.W. and A.C. carried out the main research task and wrote the manuscript. J.W. proposed the original idea and contributed to the revision of the obtained results and of the whole manuscript. Z.S., X.L. (Xiao Lin), L.S. performed utilization on-site. W.L., X.L. (Xiaotian Liu), H.L., Y.S. and Y.L. performed the investigation. All authors have read and agreed to the published version of the manuscript.

Funding: This research was funded by the Shanghai Municipal Science and Technology Project (18DZ1201301; 19DZ1200900); Xiamen Road and Bridge Group (XM2017-TZ0151; XM2017-TZ0117); the project of Key Laboratory of Impact and Safety Engineering (Ningbo University), Ministry of Education (CJ202101); Shanghai Municipal Science and Technology Major Project (2021SHZDZX0100) and the Fundamental Research Funds for the Central Universities; Key Laboratory of Land Subsidence Monitoring and Prevention, Ministry of Natural Resources of the People's Republic of China (No. KLLSMP202101); Suzhou Rail Transit Line 1 Co. Ltd. (SURT01YJ1S10002); China Railway 15 Bureau Group Co. Ltd. (CR15CG-XLDYH7-2019-GC01); the National Natural Science Foundation of China (grant No. 41907230).

Data Availability Statement: The data presented in this study are available on request from the corresponding author.

Conflicts of Interest: The authors declare no conflict of interest.

\section{References}

1. Cui, J.Q.; Nelson, J.D. Underground transport: An overview. Tunn. Undergr. Space Technol. 2019, 87, 122-126. [CrossRef]

2. Hamidreza, V.; Fatemeh, M.; Amir, R. Modified dynamic stress concentration factor for twin tunnels using a novel approach of FEM-scattering. Tunn. Undergr. Space Technol. 2017, 70, 30-41. [CrossRef]

3. Wang, H.N.; Zeng, G.S.; Sutilt, S.; Jiang, M.G.; Wu, L. Analytical solutions of stresses and displacements for deeply buried twin tunnels in viscoelastic rock. Int. J. Rock Meck. Min. 2017, 93, 13-29. [CrossRef]

4. Kim, Y.G. Application of risk analysis and assessment in tunnel design. Int. J. JCRM 2014, 5, 11-18. [CrossRef]

5. Moradi, M.R.; Farsangi, M.A.E. Application of the risk matrix method for geotechnical risk analysis and prediction of the advance rate in rock TBM tunneling. Rock Mech. Rock Eng. 2014, 47, 1951-1960. [CrossRef]

6. Shin, H.S.; Kwon, Y.C.; Jung, Y.S.; Bae, G.J.; Kim, Y.G. Methodology for quantitative hazard assessment for tunnel collapses based on case histories in Korea. Int. J. Rock Mech. Min. Sci. 2009, 46, 1072-1087. [CrossRef]

7. Li, S.C.; $\mathrm{Wu}, \mathrm{J} . ; \mathrm{Xu}, \mathrm{Z} . \mathrm{H} . ; \mathrm{Li}$, L.P. Unascertained measure model of water and mud inrush risk evaluation in karst tunnels and its engineering application. KSCE J. Civ. Eng. 2016, 21, 1170-1182. [CrossRef]

8. Li, N.; Feng, X.; Jimenez, R. Predicting rock burst hazard with incomplete data using Bayesian networks. Tunn. Undergr. Space Technol. 2017, 61, 61-70. [CrossRef]

9. Evbuomwan, P.O.; Yu, D.M. Theoretical and practical risk assessment method in tunneling. Adv. Mater. Res. 2011, 255-260, 3953-3957. [CrossRef] 
10. Zhu, K.Y. Fuzzy analytic hierarchy process: Fallacy of the popular methods. Eur. J. Oper. Res. 2014, 236, 209-217. [CrossRef]

11. Rundmo, T.; Nordfjærn, T.; Iversen, H.H.; Oltedal, S.; Jørgensen, S.H. The role of risk perception and other risk-related judgements in transportation mode use. Saf. Sci. 2011, 49, 226-235. [CrossRef]

12. Einstein, H.H.; Labreche, D.A.; Markow, M.J.; Baecher, G.B. Decision analysis applied to rock tunnel exploration. Eng. Geol. 1978, 12, 143-161. [CrossRef]

13. Einstein, H.H. Risk and risk analysis in rock engineering. Tunn. Undergr. Space Technol. 1996, 11, 141-155. [CrossRef]

14. Sturk, R.; Olsson, L.; Johansson, J. Risk and decision analysis for large underground projects, as applied to Stoekholm Ring Road tunnels. Tunn. Undergr. Space Technol. 1996, 11, 157-164. [CrossRef]

15. Reilly, J.J. The management process for complex underground and tunneling projects. Tunn. Undergr. Space Technol. 2000, 15, 31-44. [CrossRef]

16. Choi, H.H.; Cho, H.N.; Seo, J.W. Risk assessment methodology for underground construction projects. J. Constr. Eng. Undergr. Manag. 2004, 130, 258-272. [CrossRef]

17. Kuang, X.; Yi, D.Q.; Wang, C.L. Study on construction risk assessment method of deep buried tunnels. Appl. Mech. Mater. 2013, 405-408, 1305-1308. [CrossRef]

18. Li, S.C.; Wu, J. A multi-factor comprehensive risk assessment method of karst tunnels and its engineering application. Bull. Eng. Geol. Environ. 2019, 78, 1761-1776. [CrossRef]

19. Zhang, G.H.; Jiao, Y.Y.; Chen, L.B.; Wang, H.; Li, S.C. Analytical model for assessing collapse risk during mountain tunnel construction. Can. Geotechnol. J. 2016, 53, 326-342. [CrossRef]

20. Embaby, K.; Naggar, M.H.; Sharnouby, M. Response evaluation of large-span ultradeep soilsteel bridges to truck loading. Int. J. Geomech. 2021, 21, 04021186. [CrossRef]

21. Amer, W.; Lars, P.; Raid, K. FEM simulation of a full-scale loading-to-failure test of a corrugated steel culvert. Steel Compos. St. 2018, 27, 217-227. [CrossRef]

22. Maleska, T.; Beben, D. The effect of mine induced tremors on seismic response of soil-steel bridges. MATEC Web Conf. 2018, 174, 04002. [CrossRef]

23. Maleska, T.; Beben, D.; Nowacka, J. Seismic vulnerability of a soil-steel composite tunnel—Norway Tolpinrud Railway Tunnel Case Study. Tunn. Undergr. Space Technol. 2021, 110, 103808. [CrossRef]

24. Flener, E.B. Soil-Steel Interaction of Long-Span Box Culverts-Performance during Backfilling. J. Geotech. Geoenviron. Eng. 2010, 136, 823-832. [CrossRef]

25. Hong, E.S.; Lee, I.M.; Shin, H.S.; Nam, S.W.; Kong, J.S. Quantitative risk evaluation based on event tree analysis technique: Application to the design of shield TBM. Tunn. Undergr. Space Technol. 2009, 24, 269-277. [CrossRef]

26. Hyun, K.C.; Min, S.; Choi, H.; Park, J.; Lee, I.M. Risk analysis using fault-tree analysis (FTA) and analytic hierarchy process (AHP) applicable to shield TBM tunnels. Tunn. Undergr. Space Technol. 2015, 49, 121-129. [CrossRef]

27. Lyu, H.M.; Sun, W.J.; Shen, S.L.; Arulrajah, A. Flood risk assessment in metro systems of mega-cities using a GIS-based modeling approach. Sci. Total Environ. 2018, 626, 1012-1025. [CrossRef]

28. Lyu, H.M.; Shen, S.L.; Zhou, A.; Yang, J. Risk assessment of mega-city infrastructures related to land subsidence using improved trapezoidal FAHP. Sci. Total Environ. 2019, 717, 135310. [CrossRef] [PubMed]

29. Li, F.W.; Phoon, K.K.; Du, X.L.; Zhang, M.J. Improved AHP Method and Its Application in Risk Identification. J. Constr. Eng. Manag. 2013, 139, 312-320. [CrossRef]

30. Chu, H.; Xu, G.; Yasufuku, N.; Yu, Z.; Liu, P.; Wang, J. Risk assessment of water inrush in karst tunnels based on two-class fuzzy comprehensive evaluation method. Arab. J. Geosci. 2017, 10, 179. [CrossRef]

31. Balta, G.; Dikmen, I.; Birgonul, M.T. Bayesian network based decision support for predicting and mitigating delay risk in TBM tunnel projects. Autom. Constr. 2021, 129, 103819. [CrossRef]

32. Kodur, V.K.R.; Naser, M.Z. Importance factor for design of bridges against fire hazard. Eng. Struct. 2013, 54, 207-220. [CrossRef]

33. Zacchei, E.; Molina, J.L. Introducing importance factors (IFs) to estimate a dam's risk of collapse produced by seismic processes. Int. J. Disast. Risk 2021, 60, 102311. [CrossRef]

34. Deng, X.; Xu, T.; Wang, R. Risk Evaluation Model of Highway Tunnel Portal Construction Based on BP Fuzzy Neural Network. Comput. Intel. Neurosci. 2018, 2018, 8547313. [CrossRef]

35. Wang, J.; Zhong, H.; Feng, L. A model of coal mine water disaster emergency logistics risk assessment based on AHP and BP neural network. In Future Communication, Computing, Control and Management; Springer: Berlin, Germany, 2012; pp. 535-542.

36. Zhang, C.; Wu, S.; Wu, J. Study on risk assessment model of collapse during construction of mountain tunnel and its application. J. Saf. Sci. Technol. 2019, 15, 128-134. [CrossRef]

37. Lyu, H.M.; Shen, S.L.; Zhou, A.N.; Zhou, W.H. Flood risk assessment of metro systems in a subsiding environment using the interval FAHP-FCA approach. Sustain. Cities Soc. 2019, 50, 101682. [CrossRef]

38. Lyu, H.M.; Zhou, W.H.; Shen, S.L.; Zhou, A.N. Inundation risk assessment of metro system using AHP and TFN-AHP in Shenzhen. Sustain. Cities Soc. 2020, 56, 102103. [CrossRef]

39. Zheng, Q.; Lyu, H.M.; Zhou, A.; Shen, S.L. Risk assessment of geohazards along Cheng-Kun railway using fuzzy AHP incorporated into GIS. Geomat. Nat. Haz. Risk. 2021, 12, 1508-1531. [CrossRef]

40. Huseby, A.B.; Skogen, S. Dynamic risk analysis: The DynRisk concept. Int. J. Proj. Manag. 1992, 10, 160-164. [CrossRef] 
41. Yi, P.T.; Zhou, Y.; Guo, Y.J.; Li, W.W. A dynamic comprehensive evaluation method embodying development tendency. Oper. Res. Manag. Sci. 2016, 25, 175-180. [CrossRef]

42. Hu, P.; Liu, S.H.; Wang, D.G.; He, R.Y. Dynamic risk assessment for natural gas pipelines based on variable weights comprehensive theory. China Saf. Sci. J. 2012, 22, 82-88. [CrossRef]

43. Li, Y.C.; Xue, Y.D.; Li, Y.J. A new construction risk assessment method based on dynamic weight. Chin. J. Under Sp. Eng. 2017, 13, 209-215.

44. Zhao, H.; Wang, X.Q. Research on dynamic financing risk evaluation system of large-scale infrastructure projects. J. Beijing Inst. Technol. (Soc. Sci. Ed.) 2017, 19, 83-90. [CrossRef]

45. Wang, S.; Li, L.P.; Shi, S.S.; Cheng, S.; Hu, H.J.; Wen, T. Dynamic risk assessment method of collapse in mountain tunnels and application. Geotechnol. Geol. Eng. 2020, 38, 2913-2916. [CrossRef]

46. Wang, S.; Li, L.P.; Cheng, S.; Hu, H.J.; Gao, S. Dynamic risk assessment method of tunnel collapse based on attribute interval assessment model and application. Pol. J. Environ. Soc. 2020, 29, 3853-3864. [CrossRef]

47. Wang, Y.; Su, J.; Zhang, S.; Guo, S.; Zhang, P.; Du, M. A dynamic risk assessment method for deep-buried tunnels based on a bayesian network. Geofluids 2020, 2020, 8848860. [CrossRef]

48. Wu, B.; Chen, H.B.; Huang, W.; Meng, G.W. Dynamic Evaluation Method of the EW-AHP Attribute Identification Model for the Tunnel Gushing Water Disaster under Interval Conditions and Applications. Math. Probl. Eng. 2021, 2021, 6661609. [CrossRef]

49. Zheng, J.F. Research on Construction Risk Assessment of Urban Shallow-Buried Large Span Underground Excavation Engineering; Beijing Jiaotong University: Beijing, China, 2020. [CrossRef]

50. Cheng, Y.; Liu, Z.B.; Liu, S.Y.; Cai, G.J.; Tong, L.Y. Risk recognition of construction of large-span and shallow buried-highway tunnels based on analytic hierarchy process. Chin. J. Geotech. Eng. 2011, 33 (Suppl. 1), 198-202.

51. Lyu, H.M.; Sun, W.J.; Shen, S.L.; Zhou, A.N. Risk Assessment Using a New Consulting Process in Fuzzy AHP. J. Constr. Eng. Manag. 2020, 146, 04019112. [CrossRef] 